\title{
Some Existence Results for a System of Nonlinear Sequential Fractional Differential Equations with Coupled Nonseparated Boundary Conditions
}

\author{
Muath Awadalla \\ Department of Mathematics and Statistics, College of Science,King Faisal University, Hafuf, Al Ahsa 31982, Saudi Arabia \\ Correspondence should be addressed to Muath Awadalla; mawadalla@kfu.edu.sa
}

Received 20 September 2021; Revised 13 November 2021; Accepted 16 January 2022; Published 8 February 2022

Academic Editor: Abdellatif Ben Makhlouf

Copyright (c) 2022 Muath Awadalla. This is an open access article distributed under the Creative Commons Attribution License, which permits unrestricted use, distribution, and reproduction in any medium, provided the original work is properly cited.

This article concerns with the existence and uniqueness theory of solutions for sequential fractional differential system involving Caputo fractional derivatives of order $1<$ alpha, beta $<2$ with coupled nonseparated boundary conditions. The standard tools of the fixed point theory were used to establish the main results. Application is introduced to show the validity of our results.

\section{Introduction}

This article is devoted to the study of the following nonlinear sequential fractional differential equation.

$$
\left\{\begin{array}{l}
{ }^{c} D^{\alpha-1}(D+\lambda) u(t)=f\left(t, u(t), v(t),{ }^{c} D^{\alpha-1} u(t),{ }^{c} D^{\beta-1} v(t)\right), \\
{ }^{c} D^{\beta-1}(D+\mu) v(t)=g\left(t, u(t), v(t),{ }^{c} D^{\alpha-1} u(t),{ }^{c} D^{\beta-1} v(t)\right), \\
u(\eta)=\varepsilon_{1} v(T),{ }^{c} D^{\alpha-1} u(\eta)=\varepsilon_{2}{ }^{c} D^{\alpha-1} v(T), \\
v(\eta)=\delta_{1} u(T),{ }^{c} D^{\beta-1} v(\eta)=\delta_{2}{ }^{c} D^{\beta-1} u(T),
\end{array}\right.
$$

where ${ }^{c} D^{\alpha-1}$ is the fractional derivative of order in Caputo sense, and $1<\alpha, \beta \leq 2,0 \leq t \leq T 0<\eta<T, \lambda, \mu \in \mathbb{R}^{+}$, and $\varepsilon_{1}, \varepsilon_{2}, \delta_{1}, \delta_{2} \in \mathbb{R}$.

The studied equation, equation (1), is subject to boundary conditions which are coupled but nonseparated. The main goal of the article is to prove the existence of solutions to the problem defined by equation (1).

The theory of fractional differential equation subject to boundary conditions has long been of the interest of researchers. Two-point boundary conditions are common in bibliography; however, special attention is given to more complex boundary conditions such as integral and nonlocal multiple points.

Differential equations with nonlocal boundary conditions are of high importance in applied sciences. They are useful in modeling some chemical processes and in general to model a process which is located inside a defined region. On the other hand, differential equations with integral boundary conditions are appropriate for modeling problems that involve flowing, such as blood flow problem (see $[1,2])$. 
Other application examples are biology, finances, etc. (see [18-20] for illustration). Some interesting general results on boundary value problems are found in $[3,10-17]$ and [21-23]. In references [4-9, 24-26], the authors have investigated sequential fractional differential equations.

Although many articles have focused on the study of fractional differential equations with multiple boundary conditions, there is no or there are few works in the literature that studied problems with coupled nonseparated fractional boundary conditions. In this regard, we study the problem, equation (1), subject to nonseparated fractional boundary conditions.

To prove an existence and uniqueness of a solution to problem defined by equation (1), two main theorems are proposed in the work. Banach's fixed point theorem is used to establish the first theorem in which uniqueness of a solution to problem (1) is proved. The second theorem establishes existence of the solution to problem (1), using Leray-Schauder's alternative criterion. After this introductory section of this work, there are four other sections, which are organized following a hierarchical structure. The second section provides a brief review of fractional calculus definitions. The third section is dedicated to the main results of the work. The fourth section provides a numerical example to back up the theoretical results. Finally, conclusion and possible future works are discussed in the fifth section.

Notations:

$$
\begin{aligned}
& \omega_{1}(t)=\left[\left(\frac{\varepsilon_{2} \rho_{2} q_{2}}{\Delta_{1} \rho_{1}^{2} q_{1}}+\frac{\varepsilon_{2}}{\rho_{1}}\right) e^{-\lambda t}+\left(\frac{\delta_{1} \rho_{2} q_{2} \varepsilon_{1} \varepsilon_{2}}{\Delta_{1} \Delta_{2} \rho_{1}^{2} q_{1}}+\frac{\delta_{1} \varepsilon_{1}^{2}}{\Delta_{2} \rho_{2}}\right)\left(e^{-\lambda T}-e^{-\lambda \eta}\right)\right. \\
& \left.+\left(\frac{\varepsilon_{1} \varepsilon_{2} q_{2}}{\Delta_{1} \Delta_{2} \rho_{1} q_{1}}\right)\left(\delta_{1} \varepsilon_{1} e^{-\mu T}-e^{-\mu \eta}\right)+\left(\frac{\varepsilon_{1} \varepsilon_{2} q_{2} e^{-\mu T}}{\Delta_{1} \rho_{1} q_{1}}\right)-\left(\frac{\rho_{2} q_{2} \varepsilon_{2} e^{-\lambda \eta}}{\Delta_{1} \rho_{1}^{2} q_{1}}+\frac{\varepsilon_{1} e^{-\lambda \eta}}{\rho_{1}}\right)\right] \\
& \omega_{2}(t)=\left[\left(\frac{\delta_{2} \rho_{2}}{\Delta_{1} \rho_{1} q_{1}}\right) e^{-\lambda t}+\left(\frac{\varepsilon_{1} \delta_{1} \delta_{2} \rho_{2}}{\Delta_{1} \Delta_{2} \rho_{1} q_{1}}\right)\left(e^{-\lambda T}-e^{-\lambda \eta}\right)+\frac{\delta_{2} \varepsilon_{1}}{\Delta_{1} \Delta_{2} q_{1}}\left(\varepsilon_{1} e^{-\mu T}-e^{-\mu \eta}\right)+\left(\frac{\delta_{2}}{\Delta_{1} \rho_{1} q_{1}}\right)\left(\varepsilon_{1} \rho_{1} e^{-\mu T}-\rho_{2} e^{-\mu \eta}\right)\right], \\
& \omega_{3}(t)=\left[\left(\frac{\varepsilon_{1} \delta_{1} \rho_{2} q_{2}}{\Delta_{1} \Delta_{2} \rho_{1} q_{1}}+\frac{\varepsilon_{1} \delta_{1}}{\Delta_{2}}\right)\left(e^{-\lambda \eta}-e^{-\lambda T}\right)+\left(\frac{\varepsilon_{1} q_{2}}{\Delta_{1} \Delta_{2} q_{1}}\right)\left(e^{-\mu \eta}-\delta_{1} \varepsilon_{1} e^{-\mu T}\right)\right. \\
& \left.-\left(\frac{\varepsilon_{1} q_{2} e^{-\mu T}}{\Delta_{1} q_{1}}\right)-\left(\frac{\rho_{2} q_{2} e^{-\lambda \eta}}{\Delta_{1} \rho_{1} q_{1}}+e^{-\lambda \eta}\right)-\left(\frac{\rho_{2} q_{2}}{\Delta_{1} \rho_{1} q_{1}}+1\right) e^{-\lambda t}\right], \\
& \omega_{4}(t)=\left[\left(\frac{\varepsilon_{1} \delta_{1} \rho_{2}}{\Delta_{1} \Delta_{2} \rho_{1}}\right)\left(e^{-\lambda \eta}-e^{-\lambda T}\right)+\frac{\varepsilon_{1}}{\Delta_{1} \Delta_{2}}\left(e^{-\mu \eta}-\varepsilon_{1} \delta_{1} e^{-\mu T}\right)+\left(\frac{1}{\Delta_{1} \rho_{1}}\right)\left(\rho_{2} e^{-\lambda \eta}-\varepsilon_{1} \rho_{1} e^{-\mu T}\right)-\left(\frac{\rho_{2}}{\Delta_{1} \rho_{1}}\right) e^{-\lambda t}\right], \\
& \omega_{5}(t)=\left[\left(\frac{\varepsilon_{1} \varepsilon_{2} \delta_{1} \rho_{2} q_{2} \mu}{\Delta_{1} \Delta_{2} \rho_{1}^{2} q_{1}}+\frac{\varepsilon_{1} \varepsilon_{2} \delta_{1} \mu}{\Delta_{2}}\right)\left(e^{-\lambda \eta}-e^{-\lambda T}\right)+\left(\frac{\varepsilon_{1} \varepsilon_{2} q_{2} \mu}{\Delta_{1} \Delta_{2} \rho_{1} q_{1}}\right)\left(e^{-\mu \eta}-\delta_{1} \varepsilon_{1} e^{-\mu T}\right)\right. \\
& \left.-\left(\frac{\varepsilon_{1} \varepsilon_{2} q_{2} e^{-\mu T} \mu}{\Delta_{1} \Delta_{2} \rho_{1} q_{1}}\right)-\left(\frac{\varepsilon_{2} \rho_{2} q_{2} e^{-\lambda \eta} \mu}{\Delta_{1} \rho_{1}^{2} q_{1}}+\frac{\varepsilon_{2} e^{-\lambda \eta} \mu}{\rho_{1}}\right)-\left(\frac{\varepsilon_{2} \rho_{2} q_{2} \mu}{\Delta_{1} \rho_{1}^{2} q_{1}}+\frac{\varepsilon_{2} \mu}{\rho_{1}}\right) e^{-\lambda t}\right], \\
& \omega_{6}(t)=\left[\left(\frac{\varepsilon_{1} \delta_{1} \delta_{2} \rho_{2} \lambda}{\Delta_{1} \Delta_{2} \rho_{1} q_{1}}\right)\left(e^{-\lambda \eta}-e^{-\lambda T}\right)+\frac{\varepsilon_{1} \delta_{2} \lambda}{\Delta_{1} \Delta_{2} q_{1}}\left(e^{-\mu \eta}-\varepsilon_{1} \delta_{1} e^{-\mu T}\right)+\left(\frac{\delta_{2} \lambda}{\Delta_{1} \Delta_{2} \rho_{1}}\right)\left(\rho_{2} e^{-\lambda \eta}-\varepsilon_{1} \rho_{1} e^{-\mu T}\right)-\left(\frac{\rho_{2} \delta_{2} \lambda}{\Delta_{1} \Delta_{2} \rho_{1}}\right) e^{-\lambda t}\right] \text {, }
\end{aligned}
$$




$$
\begin{aligned}
& \omega_{7}(t)=\left[\left(\frac{\rho_{2} q_{2} \lambda}{\Delta_{1} \rho_{1} q_{1}}+\lambda\right) e^{-\lambda t}+\left(\frac{\varepsilon_{1} \delta_{1} \rho_{2} q_{2} \lambda}{\Delta_{1} \Delta_{2} \rho_{1} q_{1}}+\frac{\varepsilon_{1} \delta_{1} \lambda}{\Delta_{2}}\right)\left(e^{-\lambda T}-e^{-\lambda \eta}\right)+\left(\frac{\varepsilon_{1} q_{2} \lambda}{\Delta_{1} \Delta_{2} q_{1}}\right)\left(\delta_{1} \varepsilon_{1} e^{-\mu T}-e^{-\mu \eta}\right)\right. \\
& \left.+\left(\frac{\varepsilon_{1} q_{2} e^{-\mu T} \lambda}{\Delta_{1} q_{1}}\right)-\left(\frac{\rho_{2} q_{2} e^{-\lambda \eta} \lambda}{\Delta_{1} \rho_{1} q_{1}}+\lambda e^{-\lambda \eta}\right)\right] \\
& \omega_{8}(t)=\left[\left(\frac{\mu \rho_{2}}{\Delta_{1} \rho_{1}}\right) e^{-\lambda t}+\left(\frac{\varepsilon_{1} \delta_{1} \rho_{2}}{\Delta_{1} \Delta_{2} \rho_{1}}\right)\left(e^{-\lambda T}-e^{-\lambda \eta}\right)+\frac{\varepsilon_{1} \mu}{\Delta_{1} \Delta_{2}}\left(\varepsilon_{1} \delta_{1} e^{-\mu T}-e^{-\mu \eta}\right)+\left(\frac{\mu}{\Delta_{1} \rho_{1}}\right)\left(\varepsilon_{1} \rho_{1} e^{-\mu T}-\rho_{2} e^{-\lambda \eta}\right)\right], \\
& \theta_{1}(t)=\left[\left(\frac{q_{2} \varepsilon_{2}}{\Delta_{1} \rho_{1} q_{1}}\right) e^{-\mu t}+\left(\frac{\delta_{1} \rho_{2} q_{2} \varepsilon_{2}}{\Delta_{1} \Delta_{2} \rho_{1}^{2} q_{1}}+\frac{\delta_{1} \varepsilon_{1}}{\Delta_{2} \rho_{1}}\right)\left(e^{-\lambda T}-e^{-\lambda \eta}\right)+\left(\frac{q_{2} \varepsilon_{2}}{\Delta_{1} \Delta_{2} \rho_{1} q_{1}}\right)\left(\delta_{1} \varepsilon_{1} e^{-\mu T}-e^{-\mu \eta}\right)\right], \\
& \theta_{2}(t)=\left[\left(\frac{\delta_{2}}{\Delta_{1} q_{1}}\right) e^{-\mu t}+\frac{\delta_{2}}{\Delta_{1} \Delta_{2} q_{1}}\left(\delta_{1} \varepsilon_{1} e^{-\mu T}-e^{-\mu \eta}\right)+\left(\frac{\delta_{1} \delta_{2} \rho_{2}}{\Delta_{1} \Delta_{2} \rho_{1} q_{1}}\right)\left(\delta_{1} \varepsilon_{1} e^{-\lambda T}-e^{-\lambda \eta}\right)\right], \\
& \theta_{3}(t)=\left[\left(\frac{\delta_{1} \rho_{2} q_{2}}{\Delta_{1} \Delta_{2} \rho_{1} q_{1}}+\frac{\delta_{1}}{\Delta_{2}}\right)\left(e^{-\lambda \eta}-e^{-\lambda T}\right)+\left(\frac{q_{2}}{\Delta_{1} \Delta_{2} q_{1}}\right)\left(e^{-\mu \eta}-\delta_{1} \varepsilon_{1} e^{-\mu T}\right)-\left(\frac{q_{2}}{\Delta_{1} q_{1}}\right) e^{-\mu t}\right], \\
& \theta_{4}(t)=\left[\left(\frac{\delta_{1} \rho_{2}}{\Delta_{1} \Delta_{2} \rho_{1}}\right)\left(e^{-\lambda \eta}-e^{-\lambda T}\right)+\left(\frac{1}{\Delta_{1} \Delta_{2}}\right)\left(e^{-\mu \eta}-\delta_{1} \varepsilon_{1} e^{-\mu T}\right)-\left(\frac{1}{\Delta_{1}}\right) e^{-\mu t}\right], \\
& \theta_{5}(t)=\left[\left(\frac{\mu \delta_{1} \rho_{2} q_{2} \varepsilon_{2}}{\Delta_{1} \Delta_{2} \rho_{1}^{2} q_{1}}+\frac{\delta_{1} \varepsilon_{1}}{\Delta_{2} \rho_{1}}\right)\left(e^{-\lambda \eta}-e^{-\lambda T}\right)+\left(\frac{q_{2} \varepsilon_{2}}{\Delta_{1} \Delta_{2} \rho_{1} q_{1}}\right)\left(e^{-\mu \eta}-\mu \delta_{1} \varepsilon_{1} e^{-\mu T}\right)-\left(\frac{q_{2} \varepsilon_{2} \mu}{\Delta_{1} \rho_{1} q_{1}}\right) e^{-\mu t}\right], \\
& \theta_{6}(t)=\left[\left(\frac{\lambda \delta_{1} \delta_{2} \rho_{2}}{\Delta_{1} \Delta_{2} \rho_{1} q_{1}}\right)\left(e^{-\lambda \eta}-e^{-\lambda T}\right)+\left(\frac{\lambda \delta_{2}}{\Delta_{1} \Delta_{2} q_{1}}\right)\left(e^{-\mu \eta}-\delta_{1} \rho_{2} e^{-\mu T}\right)-\left(\frac{\lambda \delta_{2}}{\Delta_{1} q_{1}}\right) e^{-\mu t}\right] \text {, } \\
& \theta_{7}(t)=\left[\left(\frac{\lambda q_{2}}{\Delta_{1} q_{1}}\right) e^{-\mu t}+\left(\frac{\lambda \delta_{1} \rho_{2} q_{2}}{\Delta_{1} \Delta_{2} \rho_{1} q_{1}}+\frac{\lambda \delta_{1}}{\Delta_{2}}\right)\left(e^{-\lambda T}-e^{-\lambda \eta}\right)+\left(\frac{\lambda q_{2}}{\Delta_{1} \Delta_{2} q_{1}}\right)\left(\delta_{1} \varepsilon_{1} e^{-\mu T}-e^{-\mu \eta}\right)\right] \text {, } \\
& \theta_{8}(t)=\left[\left(\frac{\mu}{\Delta_{1}}\right) e^{-\mu t}+\left(\frac{\mu \delta_{1} \rho_{2}}{\Delta_{1} \Delta_{2} \rho_{1}}\right)\left(e^{-\lambda T}-e^{-\lambda \eta}\right)+\left(\frac{\mu}{\Delta_{1} \Delta_{2}}\right)\left(\delta_{1} \varepsilon_{1} e^{-\mu T}-e^{-\mu \eta}\right)\right],
\end{aligned}
$$

where

$$
\begin{aligned}
\rho_{1} & =\frac{-\lambda}{\Gamma(2-\alpha)} \int_{0}^{\eta}(\eta-s)^{1-\alpha} e^{-\lambda s} \mathrm{~d} s \\
q_{1} & =\frac{-\mu}{\Gamma(2-\alpha)} \int_{0}^{\eta}(\eta-s)^{1-\beta} e^{-\mu s} \mathrm{~d} s \\
\rho_{2} & =\frac{-\mu \varepsilon_{2}}{\Gamma(2-\alpha)} \int_{0}^{T}(T-s)^{1-\alpha} e^{-\mu s} \mathrm{~d} s, \\
q_{2} & =\frac{-\lambda \delta_{2}}{\Gamma(2-\alpha)} \int_{0}^{T}(T-s)^{1-\beta} e^{-\lambda s} \mathrm{~d} s, \\
\Delta_{1} & =1-\frac{\rho_{2} q_{2}}{\rho_{1} q_{1}}, \text { and } \Delta_{2}=1-\varepsilon_{1} \delta_{1}, \\
M_{1} & =\left[\left(\frac{\delta_{1} \varepsilon_{1}^{2}}{\Delta_{2}}\right)\left(e^{-\lambda T}+1\right)+\varepsilon_{1}\right], \text { and } M_{2}=\left[-\left(\frac{\delta_{1} \varepsilon_{1}}{\Delta_{2}}\right)\left(e^{-\lambda T}+1\right)-1\right], \\
N_{1} & =\left(\frac{\delta_{1} \varepsilon_{1}}{\Delta_{2}}\right), \text { and } N_{2}=-\left(\frac{\delta_{1}}{\Delta_{2}}\right),
\end{aligned}
$$




$$
\begin{aligned}
& K_{1}(t, \tau)=\frac{1}{\Gamma(\alpha-1)} \int_{\tau}^{t} e^{-\lambda(t-r)}(r-s)^{\alpha-2} \mathrm{~d} r, \int_{0}^{t} K_{1}(t, \tau) h(\tau) \mathrm{d} \tau=\int_{0}^{t} e^{-\lambda(t-r)} I^{\alpha-1} h(r) \mathrm{d} r \\
& G_{1}(t, \tau)=\frac{1}{\Gamma(\alpha-1)} \int_{\tau}^{t} e^{-\mu(t-r)}(r-\tau)^{\beta-2} \mathrm{~d} r, \int_{0}^{t} G_{1}(t, \tau) h(\tau) \mathrm{d} \tau=\int_{0}^{t} e^{-\mu(t-r)} I^{\beta-1} h(r) \mathrm{d} r, \\
& K_{2}(t, r)=\frac{1}{\Gamma(2-\alpha)} \int_{r}^{t}(t-\tau)^{1-\alpha} K_{1}(\tau, r) \mathrm{d} \tau, G_{2}(t, r)=\frac{1}{\Gamma(2-\alpha)} \int_{r}^{t}(t-\tau)^{1-\beta} G_{1}(\tau, r) \mathrm{d} \tau .
\end{aligned}
$$

Then,

$$
\frac{1}{\Gamma(2-\alpha)} \int_{0}^{\eta}(\eta-\tau)^{1-\alpha}\left(\int_{0}^{\tau} e^{-\lambda(\tau-r)} I^{\alpha-1} \varphi(r) \mathrm{d} r\right) \mathrm{d} \tau=\int_{0}^{\eta} K_{2}(\eta, r) \varphi(r) \mathrm{d} r .
$$

In a like manner,

$$
\frac{1}{\Gamma(2-\alpha)} \int_{0}^{\eta}(\eta-\tau)^{1-\beta}\left(\int_{0}^{\tau} e^{-\mu(\tau-r)} I^{\beta-1} \psi(r) \mathrm{d} r\right) \mathrm{d} \tau=\int_{0}^{\eta} G_{2}(\eta, r) \psi(r) \mathrm{d} r .
$$

Lemma 1. Let $\phi, \psi \in C([0, T], \mathbb{R})$, then the solution of the is given by following system:

$$
\left\{\begin{array}{l}
\left({ }^{c} D^{\alpha}+\lambda^{c} D^{\alpha-1}\right) u(t)=\phi(t), \\
\left({ }^{c} D^{\beta}+\mu^{c} D^{\beta-1}\right) v(t)=\psi(t), \\
u(\eta)=\varepsilon_{1} v(T),{ }^{c} D^{\alpha-1} u(\eta)=\varepsilon_{2}{ }^{c} D^{\alpha-1} v(T), \\
v(\eta)=\delta_{1} u(T),{ }^{c} D^{\beta-1} v(\eta)=\delta_{2}{ }^{c} D^{\beta-1} u(T),
\end{array}\right.
$$

$$
\begin{aligned}
u(t)= & \int_{0}^{t} K_{1}(t, x) \varphi(x) \mathrm{d} x+\omega_{1}(t) \int_{0}^{T} \psi(x) \mathrm{d} x+\omega_{2}(t) \int_{0}^{T} \varphi(x) \mathrm{d} x+\omega_{3}(t) \int_{0}^{\eta} \varphi(x) \mathrm{d} x+\omega_{4}(t) \int_{0}^{\eta} \psi(x) \mathrm{d} x \\
& +\omega_{5}(t) \int_{0}^{T} K_{2}(T, x) \psi(x) \mathrm{d} x+\omega_{6}(t) \int_{0}^{T} G_{2}(T, x) \varphi(x) \mathrm{d} x+\omega_{7}(t) \int_{0}^{\eta} K_{2}(\eta, x) \varphi(x) \mathrm{d} x+\omega_{8}(t) \int_{0}^{\eta} G_{2}(\eta, x) \psi(x) \mathrm{d} x \\
& +M_{1} \int_{0}^{T} G_{1}(T, x) \psi(x) \mathrm{d} x+M_{2} \int_{0}^{\eta} K_{1}(\eta, x) \varphi(x) \mathrm{d} x,
\end{aligned}
$$$$
v(t)=\int_{0}^{t} G_{1}(t, x) \psi(x) \mathrm{d} x+\theta_{1}(t) \int_{0}^{T} \psi(x) \mathrm{d} x+\theta_{2}(t) \int_{0}^{T} \varphi(x) \mathrm{d} x+\theta_{3}(t) \int_{0}^{\eta} \varphi(x) \mathrm{d} x+\theta_{4}(t) \int_{0}^{\eta} \psi(x) \mathrm{d} x
$$$$
+\theta_{5}(t) \int_{0}^{T} K_{2}(T, x) \psi(x) \mathrm{d} x+\theta_{6}(t) \int_{0}^{T} G_{2}(T, x) \varphi(x) d x+\theta_{7}(t) \int_{0}^{\eta} K_{2}(\eta, x) \varphi(x) \mathrm{d} x+\theta_{8}(t) \int_{0}^{\eta} G_{2}(\eta, x) \psi(x) \mathrm{d} x
$$

$$
+N_{1} \int_{0}^{T} G_{1}(T, x) \psi(x) \mathrm{d} x+N_{2} \int_{0}^{\eta} K_{1}(\eta, x) \varphi(x) \mathrm{d} x .
$$

Proof. Solving the sequential linear equations 


$$
\begin{aligned}
& { }^{c} D^{\alpha-1}(D+\lambda) u(t)=\phi(t), \\
& { }^{c} D^{\beta-1}(D+\mu) v(t)=\psi(t),
\end{aligned}
$$

Now, we need to find the constants $c_{0}, c_{1}, d_{0}$, and $d_{1}$. Before finding these constants, we find,

we get

$$
\begin{aligned}
& u(t)=c_{0} e^{-\lambda t}+c_{1}+\int_{0}^{t} e^{-\lambda(t-s)} I^{\alpha-1} \varphi(s) \mathrm{d} s, \\
& v(t)=d_{0} e^{-\mu t}+d_{1}+\int_{0}^{t} e^{-\mu(t-s)} I^{\beta-1} \psi(s) \mathrm{d} s .
\end{aligned}
$$

$$
\begin{aligned}
& { }^{c} D^{\alpha-1} u(t)=c_{0}-\lambda / \Gamma(2-\alpha)\left(\int_{0}^{t}(t-s)^{1-\alpha} e^{-\lambda s} d s\right)+\frac{1}{\Gamma(2-\alpha)}\left(\int_{0}^{t}(t-s)^{1-\alpha}\left(I^{\alpha-1} \varphi(s)-\lambda \int_{0}^{s} e^{-\lambda(s-\tau)} I^{\alpha-1} \varphi(\tau) \mathrm{d} \tau\right) \mathrm{d} s\right) \\
& { }^{c} D^{\beta-1} u(t)=c_{0} \frac{-\lambda}{\Gamma(2-\beta)} \int_{0}^{t}(t-s)^{1-\beta} e^{-\lambda s} \mathrm{~d} s+\frac{1}{\Gamma(2-\beta)} \int_{0}^{t}(t-s)^{1-\beta}\left(I^{\beta-1} \varphi(s)-\lambda \int_{0}^{s} e^{-\lambda(s-\tau)} I^{\beta-1} \varphi(\tau) \mathrm{d} \tau\right) \mathrm{d} s, \\
& { }^{c} D^{\alpha-1} v(t)=d_{0} \frac{-\mu}{\Gamma(2-\alpha)} \int_{0}^{t}(t-s)^{1-\alpha} e^{-\mu s} \mathrm{~d} s+\frac{1}{\Gamma(2-\alpha)} \int_{0}^{t}(t-s)^{1-\alpha}\left(I^{\alpha-1} \psi(s)-\mu \int_{0}^{s} e^{-\mu(s-\tau)} I^{\alpha-1} \psi(\tau) \mathrm{d} \tau\right) \mathrm{d} s, \\
& { }^{c} D^{\beta-1} u(t)=d_{0} \frac{-\mu}{\Gamma(2-\beta)} \int_{0}^{t}(t-s)^{1-\beta} e^{-\mu s} \mathrm{~d} s+\frac{1}{\Gamma(2-\beta)} \int_{0}^{t}(t-s)^{1-\beta}\left(I^{\beta-1} \psi(s)-\mu \int_{0}^{-\mu(s-\tau)} I^{\beta-1} \psi(\tau) \mathrm{d} \tau\right) \mathrm{d} s .
\end{aligned}
$$

The first boundary conditions $u(\eta)=\varepsilon_{1} v(T),{ }^{c} D^{\alpha-1}$ $u(\eta)=\varepsilon_{2}{ }^{c} D^{\alpha-1} v(T)$ give,

$$
\begin{gathered}
c_{0} e^{-\lambda \eta}+c_{1}+\int_{0}^{\eta} K_{1}(\eta, x) \varphi(x) \mathrm{d} x=d_{0} \varepsilon_{1} e^{-\mu T}+d_{1} \varepsilon_{1}+\varepsilon_{1} \int_{0}^{T} G_{1}(T, x) \psi(x) \mathrm{d} x, \\
c_{0} \rho_{1}+\int_{0}^{\eta} \varphi(x) \mathrm{d} x-\lambda \int_{0}^{\eta} K_{2}(\eta, x) \varphi(x) \mathrm{d} x=d_{0} \rho_{2}+\varepsilon_{2} \int_{0}^{T} \psi(x) \mathrm{d} x-\mu \varepsilon_{2} \int_{0}^{T} K_{2}(T, x) \psi(x) \mathrm{d} x .
\end{gathered}
$$

From the second boundary conditions $v(\eta)=\delta_{1} u(T),{ }^{c} D^{\beta-1} v(\eta)=\delta_{2}{ }^{c} D^{\beta-1} u(T)$, we get,

$$
\begin{gathered}
d_{0} e^{-\mu \eta}+d_{1}+\int_{0}^{\eta} G_{1}(\eta, x) \varphi(x) \mathrm{d} x=c_{0} \delta_{1} e^{-\lambda T}+c_{1} \delta_{1}+\delta_{1} \int_{0}^{T} G_{1}(T, x) \psi(x) \mathrm{d} x, \\
d_{0} q_{1}+\int_{0}^{\eta} \psi(x) \mathrm{d} x-\mu \int_{0}^{\eta} G_{2}(\eta, x) \psi(x) \mathrm{d} x=c_{0} q_{2}+\delta_{2} \int_{0}^{T} \varphi(x) \mathrm{d} x-\lambda \delta_{2} \int_{0}^{T} G_{2}(T, x) \varphi(x) \mathrm{d} x .
\end{gathered}
$$

A simultaneous solution of (15) and (17) implies, 
6

Complexity

$$
\begin{aligned}
d_{0}= & \frac{q_{2} \varepsilon_{2}}{\Delta_{1} \rho_{1} q_{1}} \int_{0}^{T} \psi(x) \mathrm{d} x+\frac{\delta_{2}}{\Delta_{1} q_{1}} \int_{0}^{T} \varphi(x) \mathrm{d} x-\frac{q_{2}}{\Delta_{1} q_{1}} \int_{0}^{\eta} \varphi(x) \mathrm{d} x-\frac{1}{\Delta_{1}} \int_{0}^{\eta} \psi(x) \mathrm{d} x \\
& -\frac{\mu q_{2} \varepsilon_{2}}{\Delta_{1} \rho_{1} q_{1}} \int_{0}^{T} K_{2}(T, x) \psi(x) \mathrm{d} x-\frac{\lambda \delta_{2}}{\Delta_{1} q_{1}} \int_{0}^{T} G_{2}(T, x) \varphi(x) \mathrm{d} x \\
& +\frac{\lambda q_{2}}{\Delta_{1} q_{1}} \int_{0}^{\eta} K_{2}(\eta, x) \varphi(x) \mathrm{d} x+\frac{\mu}{\Delta_{1}} \int_{0}^{\eta} G_{2}(\eta, x) \psi(x) \mathrm{d} x, \Delta_{1} \neq 0, \\
c_{0}= & \left.\frac{\rho_{2} q_{2} \varepsilon_{2}}{\Delta_{1} \rho_{1}^{2} q_{1}}+\frac{\varepsilon_{2}}{\rho_{1}}\right) \int_{0}^{T} \psi(x) \mathrm{d} x+\frac{\rho_{2} \delta_{2}}{\Delta_{1} \rho_{1} q_{1}} \int_{0}^{T} \varphi(x) \mathrm{d} x-\left(\frac{\rho_{2} q_{2}}{\Delta_{1} \rho_{1} q_{1}}+1\right) \int_{0}^{\eta} \varphi(x) \mathrm{d} x-\frac{\rho_{2}}{\Delta_{1} \rho_{1}} \int_{0}^{\eta} \psi(x) \mathrm{d} x \\
& -\left(\frac{\mu \rho_{2} q_{2} \varepsilon_{2}}{\Delta_{1} \rho_{1}^{2} q_{1}}+\frac{\mu \varepsilon_{2}}{\rho_{1}}\right) \int_{0}^{T} K_{2}(T, x) \psi(x) \mathrm{d} x-\frac{\lambda \rho_{2} \delta_{2}}{\Delta_{1} \rho_{1} q_{1}} \int_{0}^{T} G_{2}(T, x) \varphi(x) \mathrm{d} x \\
& +\left(\frac{\lambda \rho_{2} q_{2}}{\Delta_{1} \rho_{1} q_{1}}+\lambda\right) \int_{0}^{\eta} K_{2}(\eta, x) \varphi(x) \mathrm{d} x+\frac{\mu \rho_{2}}{\Delta_{1} \rho_{1}} \int_{0}^{\eta} G_{2}(\eta, x) \psi(x) \mathrm{d} x .
\end{aligned}
$$

Next, we substitute (18) and (19) into (14) and (16) and solve the result system of the equations; this leads to,

$$
\begin{aligned}
d_{1}= & {\left[\left(\frac{\delta_{1} \rho_{2} q_{2} \varepsilon_{2}}{\Delta_{1} \Delta_{2} \rho_{1}^{2} q_{1}}+\frac{\delta_{1} \varepsilon_{1}}{\Delta_{2} \rho_{1}}\right)\left(e^{-\lambda T}-e^{-\lambda \eta}\right)+\left(\frac{q_{2} \varepsilon_{2}}{\Delta_{1} \Delta_{2} \rho_{1} q_{1}}\right)\left(\delta_{1} \varepsilon_{1} e^{-\mu T}-e^{-\mu \eta}\right)\right] \int_{0}^{T} \psi(x) \mathrm{d} x } \\
& +\left[\frac{\delta_{2}}{\Delta_{1} \Delta_{2} q_{1}}\left(\delta_{1} \varepsilon_{1} e^{-\mu T}-e^{-\mu \eta}\right)+\left(\frac{\delta_{1} \delta_{2} \rho_{2}}{\Delta_{1} \Delta_{2} \rho_{1} q_{1}}\right)\left(\delta_{1} \varepsilon_{1} e^{-\lambda T}-e^{-\lambda \eta}\right)\right] \int_{0}^{T} \varphi(x) \mathrm{d} x \\
& +\left[\left(\frac{\delta_{1} \rho_{2} q_{2}}{\Delta_{1} \Delta_{2} \rho_{1} q_{1}}+\frac{\delta_{1}}{\Delta_{2}}\right)\left(e^{-\lambda \eta}-e^{-\lambda T}\right)+\left(\frac{q_{2}}{\Delta_{1} \Delta_{2} q_{1}}\right)\left(e^{-\mu \eta}-\delta_{1} \varepsilon_{1} e^{-\mu T}\right)\right] \int_{0}^{\eta} \varphi(x) \mathrm{d} x \\
& +\left[\left(\frac{\delta_{1} \rho_{2}}{\Delta_{1} \Delta_{2} \rho_{1}}\right)\left(e^{-\lambda \eta}-e^{-\lambda T}\right)+\left(\frac{1}{\Delta_{1} \Delta_{2}}\right)\left(e^{-\mu \eta}-\delta_{1} \varepsilon_{1} e^{-\mu T}\right)\right] \int_{0}^{\eta} \psi(x) \mathrm{d} x \\
& +\left[\left(\frac{\mu \delta_{1} \rho_{2} q_{2} \varepsilon_{2}}{\Delta_{1} \Delta_{2} \rho_{1}^{2} q_{1}}+\frac{\delta_{1} \varepsilon_{1}}{\Delta_{2} \rho_{1}}\right)\left(e^{-\lambda \eta}-e^{-\lambda T}\right)+\left(\frac{q_{2} \varepsilon_{2}}{\Delta_{1} \Delta_{2} \rho_{1} q_{1}}\right)\left(e^{-\mu \eta}-\mu \delta_{1} \varepsilon_{1} e^{-\mu T}\right)\right] \int_{0}^{T} K_{2}(T, x) \psi(x) \mathrm{d} x \\
& +\left[\left(\frac{\lambda \delta_{1} \delta_{2} \rho_{2}}{\Delta_{1} \Delta_{2} \rho_{1} q_{1}}\right)\left(e^{-\lambda \eta}-e^{-\lambda T}\right)+\left(\frac{\lambda \delta_{2}}{\Delta_{1} \Delta_{2} q_{1}}\right)\left(e^{-\mu \eta}-\delta_{1} \rho_{2} e^{-\mu T}\right)\right] \int_{0}^{T} G_{2}(T, x) \varphi(x) \mathrm{d} x
\end{aligned}
$$




$$
\begin{aligned}
& +\left[\left(\frac{\lambda \delta_{1} \rho_{2} q_{2}}{\Delta_{1} \Delta_{2} \rho_{1} q_{1}}+\frac{\lambda \delta_{1}}{\Delta_{2}}\right)\left(e^{-\lambda T}-e^{-\lambda \eta}\right)+\left(\frac{\lambda q_{2}}{\Delta_{1} \Delta_{2} q_{1}}\right)\left(\delta_{1} \varepsilon_{1} e^{-\mu T}-e^{-\mu \eta}\right)\right] \int_{0}^{\eta} K_{2}(\eta, x) \varphi(x) \mathrm{d} x \\
& +\left[\left(\frac{\mu \delta_{1} \rho_{2}}{\Delta_{1} \Delta_{2} \rho_{1}}\right)\left(e^{-\lambda T}-e^{-\lambda \eta}\right)+\left(\frac{\mu}{\Delta_{1} \Delta_{2}}\right)\left(\delta_{1} \varepsilon_{1} e^{-\mu T}-e^{-\mu \eta}\right)\right] \int_{0}^{\eta} G_{2}(\eta, x) \psi(x) \mathrm{d} x \\
& +\left(\frac{\delta_{1} \varepsilon_{1}}{\Delta_{2}}\right) \int_{0}^{T} G_{1}(T, x) \psi(x) \mathrm{d} x-\left(\frac{\delta_{1}}{\Delta_{2}}\right) \int_{0}^{\eta} K_{1}(\eta, x) \varphi(x) \mathrm{d} x . \\
& c_{1}=\left[\left(\frac{\delta_{1} \rho_{2} q_{2} \varepsilon_{1} \varepsilon_{2}}{\Delta_{1} \Delta_{2} \rho_{1}^{2} q_{1}}+\frac{\delta_{1} \varepsilon_{1}^{2}}{\Delta_{2} \rho_{2}}\right)\left(e^{-\lambda T}-e^{-\lambda \eta}\right)+\left(\frac{\varepsilon_{1} \varepsilon_{2} q_{2}}{\Delta_{1} \Delta_{2} \rho_{1} q_{1}}\right)\left(\delta_{1} \varepsilon_{1} e^{-\mu T}-e^{-\mu \eta}\right)\right. \\
& \left.+\left(\frac{\varepsilon_{1} \varepsilon_{2} q_{2} e^{-\mu T}}{\Delta_{1} \rho_{1} q_{1}}\right)-\left(\frac{\rho_{2} q_{2} \varepsilon_{2} e^{-\lambda \eta}}{\Delta_{1} \rho_{1}^{2} q_{1}}+\frac{\varepsilon_{1} e^{-\lambda \eta}}{\rho_{1}}\right)\right] \int_{0}^{T} \psi(x) \mathrm{d} x \\
& +\left[\left(\frac{\varepsilon_{1} \delta_{1} \delta_{2} \rho_{2}}{\Delta_{1} \Delta_{2} \rho_{1} q_{1}}\right)\left(e^{-\lambda T}-e^{-\lambda \eta}\right)+\frac{\delta_{2} \varepsilon_{1}}{\Delta_{1} \Delta_{2} q_{1}}\left(\varepsilon_{1} e^{-\mu T}-e^{-\mu \eta}\right)+\left(\frac{\delta_{2}}{\Delta_{1} \rho_{1} q_{1}}\right)\left(\varepsilon_{1} \rho_{1} e^{-\mu T}-\rho_{2} e^{-\mu \eta}\right)\right] \int_{0}^{T} \varphi(x) \mathrm{d} x \\
& +\left[\left(\frac{\varepsilon_{1} \delta_{1} \rho_{2} q_{2}}{\Delta_{1} \Delta_{2} \rho_{1} q_{1}}+\frac{\varepsilon_{1} \delta_{1}}{\Delta_{2}}\right)\left(e^{-\lambda \eta}-e^{-\lambda T}\right)+\left(\frac{\varepsilon_{1} q_{2}}{\Delta_{1} \Delta_{2} q_{1}}\right)\left(e^{-\mu \eta}-\delta_{1} \varepsilon_{1} e^{-\mu T}\right)\right. \\
& \left.-\left(\frac{\varepsilon_{1} q_{2} e^{-\mu T}}{\Delta_{1} q_{1}}\right)-\left(\frac{\rho_{2} q_{2} e^{-\lambda \eta}}{\Delta_{1} \rho_{1} q_{1}}+e^{-\lambda \eta}\right)\right] \int_{0}^{\eta} \varphi(x) \mathrm{d} x \\
& +\left[\left(\frac{\varepsilon_{1} \delta_{1} \rho_{2}}{\Delta_{1} \Delta_{2} \rho_{1}}\right)\left(e^{-\lambda \eta}-e^{-\lambda T}\right)+\frac{\varepsilon_{1}}{\Delta_{1} \Delta_{2}}\left(e^{-\mu \eta}-\varepsilon_{1} \delta_{1} e^{-\mu T}\right)+\left(\frac{1}{\Delta_{1} \rho_{1}}\right)\left(\rho_{2} e^{-\lambda \eta}-\varepsilon_{1} \rho_{1} e^{-\mu T}\right)\right] \int_{0}^{\eta} \psi(x) \mathrm{d} x \\
& +\left[\left(\frac{\varepsilon_{1} \varepsilon_{2} \delta_{1} \rho_{2} q_{2} \mu}{\Delta_{1} \Delta_{2} \rho_{1}^{2} q_{1}}+\frac{\varepsilon_{1} \varepsilon_{2} \delta_{1} \mu}{\Delta_{2}}\right)\left(e^{-\lambda \eta}-e^{-\lambda T}\right)+\left(\frac{\varepsilon_{1} \varepsilon_{2} q_{2} \mu}{\Delta_{1} \Delta_{2} \rho_{1} q_{1}}\right)\left(e^{-\mu \eta}-\delta_{1} \varepsilon_{1} e^{-\mu T}\right)\right. \\
& \left.-\left(\frac{\varepsilon_{1} \varepsilon_{2} q_{2} e^{-\mu T} \mu}{\Delta_{1} \Delta_{2} \rho_{1} q_{1}}\right)-\left(\frac{\varepsilon_{2} \rho_{2} q_{2} e^{-\lambda \eta} \mu}{\Delta_{1} \rho_{1}^{2} q_{1}}+\frac{\varepsilon_{2} e^{-\lambda \eta} \mu}{\rho_{1}}\right)\right] \int_{0}^{T} K_{2}(T, x) \psi(x) \mathrm{d} x \\
& +\left[\left(\frac{\varepsilon_{1} \delta_{1} \delta_{2} \rho_{2} \lambda}{\Delta_{1} \Delta_{2} \rho_{1} q_{1}}\right)\left(e^{-\lambda \eta}-e^{-\lambda T}\right)+\frac{\varepsilon_{1} \delta_{2} \lambda}{\Delta_{1} \Delta_{2} q_{1}}\left(e^{-\mu \eta}-\varepsilon_{1} \delta_{1} e^{-\mu T}\right)+\left(\frac{\delta_{2} \lambda}{\Delta_{1} \Delta_{2} \rho_{1}}\right)\left(\rho_{2} e^{-\lambda \eta}-\varepsilon_{1} \rho_{1} e^{-\mu T}\right)\right] \int_{0}^{T} G_{2}(T, x) \varphi(x) \mathrm{d} x \\
& +\left[\left(\frac{\varepsilon_{1} \delta_{1} \rho_{2} q_{2} \lambda}{\Delta_{1} \Delta_{2} \rho_{1} q_{1}}+\frac{\varepsilon_{1} \delta_{1} \lambda}{\Delta_{2}}\right)\left(e^{-\lambda T}-e^{-\lambda \eta}\right)+\left(\frac{\varepsilon_{1} q_{2} \lambda}{\Delta_{1} \Delta_{2} q_{1}}\right)\left(\delta_{1} \varepsilon_{1} e^{-\mu T}-e^{-\mu \eta}\right)\right. \\
& \left.+\left(\frac{\varepsilon_{1} q_{2} e^{-\mu T} \lambda}{\Delta_{1} q_{1}}\right)-\left(\frac{\rho_{2} q_{2} e^{-\lambda \eta} \lambda}{\Delta_{1} \rho_{1} q_{1}}+\lambda e^{-\lambda \eta}\right)\right] \int_{0}^{\eta} K_{2}(\eta, x) \varphi(x) \mathrm{d} x \\
& +\left[\left(\frac{\varepsilon_{1} \delta_{1} \rho_{2}}{\Delta_{1} \Delta_{2} \rho_{1}}\right)\left(e^{-\lambda T}-e^{-\lambda \eta}\right)+\frac{\varepsilon_{1} \mu}{\Delta_{1} \Delta_{2}}\left(\varepsilon_{1} \delta_{1} e^{-\mu T}-e^{-\mu \eta}\right)+\left(\frac{\mu}{\Delta_{1} \rho_{1}}\right)\left(\varepsilon_{1} \rho_{1} e^{-\mu T}-\rho_{2} e^{-\lambda \eta}\right)\right] \int_{0}^{\eta} G_{2}(\eta, x) \psi(x) \mathrm{d} x \\
& +\left[\left(\frac{\delta_{1} \varepsilon_{1}^{2}}{\Delta_{2}}\right)\left(e^{-\lambda T}+1\right)+\varepsilon_{1}\right] \int_{0}^{T} G_{1}(T, x) \psi(x) d x+\left[-\left(\frac{\delta_{1} \varepsilon_{1}}{\Delta_{2}}\right)\left(e^{-\lambda T}+1\right)-1\right] \int_{0}^{\eta} K_{1}(\eta, x) \varphi(x) \mathrm{d} x .
\end{aligned}
$$

By finding the constants $c_{0}, c_{1}, d_{0}$, and $d_{1}$ and substituting them in (12), we get the solution, proof completed.

Lemma 2 (see [21]). For any $\phi, \omega \in C([0, T] ; \mathbb{R})$, we have | $\int_{0}^{t} K_{1}(t, \tau) \phi(\tau) d \tau-\int_{0}^{t} K_{1}(t, \tau) \quad \omega(\tau) d \tau \mid \leq\left(t^{\alpha-1} / \lambda \Gamma(\alpha)\right)$ $\left(1-e^{-\lambda t}\right)\|\phi-\omega\|,\left|\int_{0}^{t} G_{1}(t, \tau) \phi(\tau) d \tau-\int_{0}^{t} G_{1}(t, \tau) p(\tau) d \tau\right|$ $\leq\left(t^{\beta-1} / \mu \Gamma(\beta)\right) \quad\left(1-e^{-\mu t}\right)\|\phi-\omega\|, \quad \mid \int_{0}^{t} K_{2}(t, \tau) \phi(\tau) d \tau-$ $\int_{0}^{t} K_{2}(t, \tau) \omega(\tau) d \tau\left|\leq(t / \lambda)\left(1-e^{-\lambda t}\right)\|\phi-\omega\|,\right| \int_{0}^{t} G_{2}(t, \tau) \phi$ $(\tau) d \tau-\int_{0}^{t} G_{2}(t, \tau) \omega(\tau) d \tau \mid \leq(t / \mu)\left(1-e^{-\mu t}\right)\|\phi-\omega\|$.

\section{Preliminaries}

Some definitions of fractional are introduced in this section as they are required in sequel of this study.

Definition 1 (see [15]). Consider a real number $\gamma>0$; the Mittag-Leffler function with one parameter is computed as 
$E_{\gamma}(x)=\sum_{k=0}^{\infty} x^{k} / \Gamma(\gamma k+1)$, where $x \in \mathbb{R}$ and $\Gamma$ is the gamma function $\Gamma(r)=\int_{0}^{\infty} e^{-t} s^{r-1} \mathrm{~d} s, \forall r>0$.
Definition 2 (see [15]). Given $\alpha \in \mathbb{R}^{+}$demonstrating the order of the derivative, the Caputo fractional derivative of order $\alpha$ of a function $\varphi:[0,+\infty] \longrightarrow \mathbb{R}$ is given by

$$
\left({ }^{C} D_{0^{+}}^{\alpha} \varphi\right)(\tau)= \begin{cases}\int_{0}^{\tau} \frac{(\tau-r)^{p-\alpha-1} h^{(p)}(r)}{\Gamma(n-\alpha)} \mathrm{d} r, & p-1<\alpha<p, p \in \mathbb{N}^{*}, \\ h^{(p)}(\tau), & \alpha \in \mathbb{N} .\end{cases}
$$

\section{Main Results}

Given $U_{\alpha-1}=\left\{u \mid u \in C([0, T], \mathbb{R})\right.$ and ${ }^{c} D^{\alpha-1} u \in C([0, T]$, $\mathbb{R})\}$, and we set $\|u\|_{\alpha-1}=\|u\|_{\alpha-1}+\left\|{ }^{c} D^{\alpha-1} u\right\|_{\alpha-1}=\sup _{0 \leq t \leq T}$ $|u(t)|+\sup _{0 \leq t \leq T}\left|c D^{\alpha-1} u(t)\right|$, it is known that $\left(U_{\alpha-1},\|\cdot\|_{\alpha-1}\right)$ is a Banach space, see [22].

Also, let $\quad V_{\beta-1}=\left\{v \mid v \in C([0, T], \mathbb{R})\right.$ and ${ }^{c} D^{\beta-1} v \in C$ $([0, T], \mathbb{R})\}$, and the norm $\|v\|_{\beta-1}=\|v\|_{\beta-1}+\left\|{ }^{c} D^{\alpha-1} v\right\|$ ${ }_{\beta-1}=\sup _{0 \leq t \leq T}|v(t)|+\sup _{0 \leq t \leq T}\left|c D^{\beta-1} v(t)\right|$, again $\left(V_{\beta-1}, \|\right.$. $\left.\|_{\beta-1}\right)$ is a Banach space.

It is well known that the product space $\Omega=\left(U_{\alpha-1} \times\right.$ $\left.V_{\beta-1},\|\cdot\|_{\alpha-1, \beta-1}\right)$ is a Banach space with the norm,

$$
\|(u, v)\|_{\alpha-1, \beta-1}=\|u\|_{\alpha-1}+\|v\|_{\beta-1} .
$$

Now, substituting $\varphi, \psi \quad$ by $f\left(\cdot, u(\cdot), v(\cdot),{ }^{c} D^{\alpha-1} u\right.$ $\left.(\cdot),{ }^{c} D^{\beta-1} v(\cdot)\right), g\left(\cdot, u(\cdot), v(\cdot),{ }^{c} D^{\alpha-1} u(\cdot),{ }^{c} D^{\beta-1} v(\cdot)\right) \quad$ in Lemma 1 respectively. Then, we define $\mathscr{F}: \Omega \longrightarrow \Omega$ allied to problem (1) as,

$$
\mathscr{F}:=\left(\begin{array}{l}
\mathscr{F}_{1}(u, v) \\
\mathscr{F}_{2}(u, v)
\end{array}\right),
$$

where

$$
\begin{aligned}
\mathscr{F}_{1}(u, v)(t)= & \int_{0}^{t} K_{1}(t, \tau) f\left(\tau, u(\tau), v(\tau),{ }^{c} D^{\alpha-1} u(\tau),{ }^{c} D^{\beta-1} v(\tau)\right) \mathrm{d} \tau \\
& +\omega_{1}(t) \int_{0}^{T} g\left(\tau, u(\tau), v(\tau),{ }^{c} D^{\alpha-1} u(\tau),{ }^{c} D^{\beta-1} v(\tau)\right) \mathrm{d} \tau+\omega_{2}(t) \int_{0}^{T} f\left(\tau, u(\tau), v(\tau),{ }^{c} D^{\alpha-1} u(\tau),{ }^{c} D^{\beta-1} v(\tau)\right) \mathrm{d} \tau \\
& +\omega_{3}(t) \int_{0}^{\eta} f\left(\tau, u(\tau), v(\tau),{ }^{c} D^{\alpha-1} u(\tau),{ }^{c} D^{\beta-1} v(\tau)\right) \mathrm{d} \tau+\omega_{4}(t) \int_{0}^{\eta} g\left(\tau, u(\tau), v(\tau),{ }^{c} D^{\alpha-1} u(\tau),{ }^{c} D^{\beta-1} v(\tau)\right) \mathrm{d} \tau \\
& +\omega_{5}(t) \int_{0}^{T} K_{2}(T, \tau) f\left(\tau, u(\tau), v(\tau),{ }^{c} D^{\alpha-1} u(\tau),{ }^{c} D^{\beta-1} v(\tau)\right) \mathrm{d} \tau+\omega_{6}(t) \int_{0}^{T} G_{2}(T, \tau) f\left(\tau, u(\tau), v(\tau),{ }^{c} D^{\alpha-1} u(\tau),{ }^{c} D^{\beta-1} v(\tau)\right) \mathrm{d} \tau \\
& +\omega_{7}(t) \int_{0}^{\eta} K_{2}(\eta, \tau) f\left(\tau, u(\tau), v(\tau),{ }^{c} D^{\alpha-1} u(\tau),{ }^{c} D^{\beta-1} v(\tau)\right) \mathrm{d} \tau+\omega_{8}(t) \int_{0}^{\eta} G_{2}(\eta, \tau) g\left(\tau, u(\tau), v(\tau),{ }^{c} D^{\alpha-1} u(\tau),{ }^{c} D^{\beta-1} v(\tau)\right) \mathrm{d} \tau+ \\
& +M_{1} \int_{0}^{T} G_{1}(T, \tau) g\left(\tau, u(\tau), v(\tau),{ }^{c} D^{\alpha-1} u(\tau),{ }^{c} D^{\beta-1} v(\tau)\right) \mathrm{d} \tau+M_{2} \int_{0}^{\eta} K_{1}(\eta, \tau) f\left(\tau, u(\tau), v(\tau),{ }^{c} D^{\alpha-1} u(\tau),{ }^{c} D^{\beta-1} v(\tau)\right) d \tau . \\
\mathscr{F}_{2}(u, v)(t)= & \int_{0}^{t} G_{1}(t, \tau) g\left(\tau, u(\tau), v(\tau),{ }^{c} D^{\alpha-1} u(\tau),{ }^{c} D^{\beta-1} v(\tau)\right) \mathrm{d} \tau \\
& +\theta_{1}(t) \int_{0}^{T} g\left(\tau, u(\tau), v(\tau),{ }^{c} D^{\alpha-1} u(\tau),{ }^{c} D^{\beta-1} v(\tau)\right) \mathrm{d} \tau+\theta_{2}(t) \int_{0}^{T} f\left(\tau, u(\tau), v(\tau),{ }^{c} D^{\alpha-1} u(\tau),{ }^{c} D^{\beta-1} v(\tau)\right) \mathrm{d} \tau \\
& +\theta_{3}(t) \int_{0}^{\eta} f\left(\tau, u(\tau), v(\tau),{ }^{c} D^{\alpha-1} u(\tau),{ }^{c} D^{\beta-1} v(\tau)\right) \mathrm{d} \tau+\theta_{4}(t) \int_{0}^{\eta} g\left(\tau, u(\tau), v(\tau),{ }^{c} D^{\alpha-1} u(\tau),{ }^{c} D^{\beta-1} v(\tau)\right) \mathrm{d} \tau \\
& +\theta_{5}(t) \int_{0}^{T} K_{2}(T, \tau) g\left(\tau, u(\tau), v(\tau),{ }^{c} D^{\alpha-1} u(\tau),{ }^{c} D^{\beta-1} v(\tau)\right) \mathrm{d} \tau+\theta_{6}(t) \int_{0}^{T} G_{2}(T, \tau) f\left(x, u(x), v(x),{ }^{c} D^{\alpha-1} u(x),{ }^{c} D^{\beta-1} v(x)\right) \mathrm{d} x \\
& +\theta_{7}(t) \int_{0}^{\eta} K_{2}(\eta, \tau) f\left(\tau, u(\tau), v(\tau),{ }^{c} D^{\alpha-1} u(\tau),{ }^{c} D^{\beta-1} v(\tau)\right) \mathrm{d} \tau \mathrm{d} x+\theta_{8}(t) \int_{0}^{\eta} G_{2}(\eta, \tau) g\left(\tau, u(\tau), v(\tau),{ }^{c} D^{\alpha-1} u(\tau),{ }^{c} D^{\beta-1} v(\tau)\right) \mathrm{d} \tau \\
& +N_{1} \int_{0}^{T} G_{1}(T, \tau) g\left(\tau, u(\tau), v(\tau),{ }^{c} D^{\alpha-1} u(\tau),{ }^{c} D^{\beta-1} v(\tau)\right) \mathrm{d} \tau+N_{2} \int_{0}^{\eta} K_{1}(\eta, \tau) f\left(\tau, u(\tau), v(\tau),{ }^{c} D^{\alpha-1} u(\tau),{ }^{c} D^{\beta-1} v(\tau)\right) \mathrm{d} \tau .
\end{aligned}
$$

Observe that, 


$$
\begin{aligned}
& \left({ }^{c} D^{\alpha-1} \mathscr{F}_{1}(u, v)\right)(t)=\int_{0}^{t} f\left(\tau, u(\tau), v(\tau),{ }^{c} D^{\alpha-1} u(\tau),{ }^{c} D^{\beta-1} v(\tau)\right) \mathrm{d} \tau-\lambda \int_{0}^{t} K_{2}(t, \tau) f\left(\tau, u(\tau), v(\tau),{ }^{c} D^{\alpha-1} u(\tau),{ }^{c} D^{\beta-1} v(\tau)\right) \mathrm{d} \tau \\
& +\left({ }^{c} D^{\alpha-1} \omega_{1}\right)(t) \int_{0}^{T} g\left(\tau, u(\tau), v(\tau),{ }^{c} D^{\alpha-1} u(\tau),{ }^{c} D^{\beta-1} v(\tau)\right) \mathrm{d} \tau+\left({ }^{c} D^{\alpha-1} \omega_{2}\right)(t) \int_{0}^{T} f\left(\tau, u(\tau), v(\tau),{ }^{c} D^{\alpha-1} u(\tau),{ }^{c} D^{\beta-1} v(\tau)\right) \mathrm{d} \tau \\
& +\left({ }^{c} D^{\alpha-1} \omega_{3}\right)(t) \int_{0}^{\eta} f\left(\tau, u(\tau), v(\tau),{ }^{c} D^{\alpha-1} u(\tau),{ }^{c} D^{\beta-1} v(\tau)\right) \mathrm{d} \tau+\left({ }^{c} D^{\alpha-1} \omega_{4}\right)(t) \int_{0}^{\eta} g\left(\tau, u(\tau), v(\tau),{ }^{c} D^{\alpha-1} u(\tau),{ }^{c} D^{\beta-1} v(\tau)\right) \mathrm{d} \tau \\
& +\left({ }^{c} D^{\alpha-1} \omega_{5}\right)(t) \int_{0}^{T} K_{2}(T, \tau) g\left(\tau, u(\tau), v(\tau),{ }^{c} D^{\alpha-1} u(\tau),{ }^{c} D^{\beta-1} v(\tau)\right) \mathrm{d} \tau+\left({ }^{c} D^{\alpha-1} \omega_{6}\right)(t) \int_{0}^{T} G_{2}(T, \tau) f\left(\tau, u(\tau), v(\tau),{ }^{c} D^{\alpha-1} u(\tau),{ }^{c} D^{\beta-1} v(\tau)\right) \mathrm{d} \tau \\
& +\left({ }^{c} D^{\alpha-1} \omega_{7}\right)(t) \int_{0}^{\eta} K_{2}(\eta, \tau) f\left(\tau, u(\tau), v(\tau),{ }^{c} D^{\alpha-1} u(\tau),{ }^{c} D^{\beta-1} v(\tau)\right) \mathrm{d} \tau+\left({ }^{c} D^{\alpha-1} \omega_{8}\right)(t) \int_{0}^{\eta} G_{2}(\eta, \tau) g\left(\tau, u(\tau), v(\tau),{ }^{c} D^{\alpha-1} u(\tau),{ }^{c} D^{\beta-1} v(\tau)\right) \mathrm{d} \tau . \\
& \left({ }^{c} D^{\beta-1} \mathscr{F}_{2}(u, v)\right)(t)=\int_{0}^{t} g\left(\tau, u(\tau), v(\tau),{ }^{c} D^{\alpha-1} u(\tau),{ }^{c} D^{\beta-1} v(\tau)\right) \mathrm{d} \tau-\mu \int_{0}^{t} G_{2}(t, \tau) g\left(\tau, u(\tau), v(\tau),{ }^{c} D^{\alpha-1} u(\tau),{ }^{c} D^{\beta-1} v(\tau)\right) \mathrm{d} \tau \\
& +\left({ }^{c} D^{\beta-1} \theta_{1}\right)(t) \int_{0}^{T} g\left(\tau, u(\tau), v(\tau),{ }^{c} D^{\alpha-1} u(\tau),{ }^{c} D^{\beta-1} v(\tau)\right) \mathrm{d} \tau+\left({ }^{c} D^{\beta-1} \theta_{2}\right)(t) \int_{0}^{T} f\left(\tau, u(\tau), v(\tau),{ }^{c} D^{\alpha-1} u(\tau),{ }^{c} D^{\beta-1} v(\tau)\right) \mathrm{d} \tau \\
& +\left({ }^{c} D^{\beta-1} \theta_{3}\right)(t) \int_{0}^{\eta} f\left(\tau, u(\tau), v(\tau),{ }^{c} D^{\alpha-1} u(\tau),{ }^{c} D^{\beta-1} v(\tau)\right) \mathrm{d} \tau+\left({ }^{c} D^{\beta-1} \theta_{4}\right)(t) \int_{0}^{\eta} g\left(\tau, u(\tau), v(\tau),{ }^{c} D^{\alpha-1} u(\tau),{ }^{c} D^{\beta-1} v(\tau)\right) \mathrm{d} \tau \\
& +\left({ }^{c} D^{\beta-1} \theta_{5}\right)(t) \int_{0}^{T} K_{2}(T, \tau) g\left(\tau, u(\tau), v(\tau),{ }^{C} D^{\alpha-1} u(\tau),{ }^{c} D^{\beta-1} v(\tau)\right) d \tau+\left({ }^{c} D^{\beta-1} \theta_{6}\right)(t) \int_{0}^{T} G_{2}(T, \tau) f\left(\tau, u(\tau), v(\tau),{ }^{c} D^{\alpha-1} u(\tau),{ }^{C} D^{\beta-1} v(\tau)\right) \mathrm{d} \tau \\
& +\left({ }^{c} D^{\beta-1} \theta_{7}\right)(t) \int_{0}^{\eta} K_{2}(\eta, \tau) f\left(\tau, u(\tau), v(\tau),{ }^{c} D^{\alpha-1} u(\tau),{ }^{c} D^{\beta-1} v(\tau)\right) \mathrm{d} \tau+\left({ }^{c} D^{\beta-1} \theta_{8}\right)(t) \int_{0}^{\eta} G_{2}(\eta, \tau) g\left(\tau, u(\tau), v(\tau),{ }^{c} D^{\alpha-1} u(\tau),{ }^{c} D^{\beta-1} v(\tau)\right) \mathrm{d} \tau .
\end{aligned}
$$

For computational convenience, we set,

where

$$
\begin{aligned}
Q & =\mathscr{Q}_{1}+\mathscr{Q}_{2}, \mathscr{Q}^{*}=\mathscr{Q}_{1}^{*}+\mathscr{Q}_{2}^{*}, \\
\mathscr{R} & =\mathscr{R}_{1}+\mathscr{R}_{2}, \mathscr{R}^{*}=\mathscr{R}_{1}^{*}+\mathscr{R}_{2}^{*},
\end{aligned}
$$

$$
\begin{aligned}
Q_{1} & =\left[\sup _{0 \leq t \leq T} \int_{0}^{t}\left|K_{1}(t, x)\right| \mathrm{d} x+T\left\|\omega_{2}\right\|+\eta\left\|\omega_{3}\right\|+\left\|\omega_{6}\right\| \int_{0}^{T}\left|G_{2}(T, x)\right| \mathrm{d} x+\left\|\omega_{7}\right\| \int_{0}^{\eta}\left|K_{2}(\eta, x)\right| \mathrm{d} x+\left|M_{2}\right| \int_{0}^{\eta}\left|K_{1}(\eta, x)\right| \mathrm{d} x\right], \\
\mathcal{Q}_{1}^{*}= & {\left[T\left\|\omega_{1}\right\|+\eta\left\|\omega_{4}\right\|+\left\|\omega_{5}\right\| \int_{0}^{T}\left|K_{2}(T, x)\right| \mathrm{d} x+\left\|\omega_{8}\right\| \int_{0}^{\eta}\left|G_{2}(\eta, x)\right| \mathrm{d} x+\left|M_{1}\right| \int_{0}^{T}\left|G_{1}(T, x)\right| \mathrm{d} x\right], } \\
Q_{2}= & {\left[\sup _{0 \leq t \leq T} \int_{0}^{t} \mathrm{~d} x+\lambda \int_{0}^{t}\left|K_{2}(t, x)\right| \mathrm{d} x+T\left\|{ }^{c} D^{\alpha-1} \omega_{2}\right\|+\eta\left\|c D^{\alpha-1} \omega_{3}\right\|+\left\|{ }^{c} D^{\alpha-1} \omega_{6}\right\| \int_{0}^{T}\left|G_{2}(T, x)\right| \mathrm{d} x+\left\|{ }^{c} D^{\alpha-1} \omega_{7}\right\| \int_{0}^{\eta}\left|K_{2}(\eta, x)\right| \mathrm{d} x\right], } \\
Q_{2}^{*}= & {\left[T\left\|{ }^{c} D^{\alpha-1} \omega_{1}\right\|+\eta\|\|^{c} D^{\alpha-1} \omega_{4}\|+\|{ }^{c} D^{\alpha-1} \omega_{5}\left\|\int_{0}^{T}\left|K_{2}(T, x)\right| \mathrm{d} x+\right\|{ }^{c} D^{\alpha-1} \omega_{8} \| \int_{0}^{\eta}\left|G_{2}(\eta, x)\right| \mathrm{d} x\right], } \\
\mathscr{R}_{1}= & {\left[\left\|\theta_{2}\right\| \int_{0}^{T} d x+\left\|\theta_{3}\right\| \int_{0}^{\eta} d x+\left\|\theta_{6}\right\| \int_{0}^{T}\left|G_{2}(T, x)\right| d x++\left\|\theta_{7}\right\| \int_{0}^{\eta}\left|K_{2}(\eta, x)\right| d x+\left|N_{2}\right| \int_{0}^{\eta}\left|K_{1}(\eta, x)\right| d x\right], } \\
\mathscr{R}_{1}^{*}= & {\left[\sup _{0 \leq t \leq T} \int_{0}^{t}\left|G_{1}(t, x)\right| d x+\left\|\theta_{1}\right\| \int_{0}^{T} d x+\left\|\theta_{4}\right\| \int_{0}^{\eta} d x+\left\|\theta_{5}\right\| \int_{0}^{T}\left|K_{2}(T, x)\right| d x+\left\|\theta_{8}\right\| \int_{0}^{\eta}\left|G_{2}(\eta, x)\right| d x+\left|N_{1}\right| \int_{0}^{T}\left|G_{1}(T, x)\right| d x\right], } \\
\mathscr{R}_{2}= & {\left[\left\|c D^{\beta-1} \theta_{2}\right\| \int_{0}^{T} \mathrm{~d} x+\left\|{ }^{c} D^{\beta-1} \theta_{3}\right\| \int_{0}^{\eta} \mathrm{d} x++\left\|{ }^{c} D^{\beta-1} \theta_{6}\right\| \int_{0}^{T}\left|G_{2}(T, x)\right| \mathrm{d} x+\|\|^{c} D^{\beta-1} \theta_{6} \| \int_{0}^{\eta}\left|K_{2}(\eta, x)\right| \mathrm{d} x\right], } \\
\mathscr{R}_{2}^{*}= & {\left[\sup _{0 \leq t \leq T} \int_{0}^{t} \mathrm{~d} x+\mu \sup \int_{0 \leq t \leq T}^{t}\left|G_{2}(t, x)\right| \mathrm{d} x+\left\|{ }^{c} D^{\beta-1} \theta_{1}\right\| \int_{0}^{T} \mathrm{~d} x+\left\|{ }^{c} D^{\beta-1} \theta_{4}\right\| \int_{0}^{\eta} \mathrm{d} x+\left\|{ }^{c} D^{\beta-1} \theta_{5}\right\| \int_{0}^{T}\left|K_{2}(T, x)\right| \mathrm{d} x+\left\|{ }^{c} D^{\beta-1} \theta_{8}\right\| \int_{0}^{\eta}\left|G_{2}(\eta, x)\right| \mathrm{d} x\right] . }
\end{aligned}
$$


To state the existence result for problem (1), we set the following assumptions:
(A1) The functions $f, g:[0, T] \times \mathbb{R}^{4} \longrightarrow \mathbb{R}$ are jointly continuous.

(A2) $\exists L_{f}, L_{g}>0$ such that

$$
\begin{aligned}
& \left|f\left(t, u_{1}, v_{1}, \overline{u_{1}}, \overline{v_{1}}\right)-f\left(t, u_{2}, v_{2}, \overline{u_{2}}, \overline{v_{2}}\right)\right| \leq L_{f}\left(\left|u_{1}-u_{2}\right|+\left|\overline{u_{1}}-\overline{u_{2}}\right|+\left|v_{1}-v_{2}\right|+\left|\overline{v_{1}}-\overline{v_{2}}\right|\right), \\
& \left|g\left(t, u_{1}, v_{1}, \overline{u_{1}}, \overline{v_{1}}\right)-g\left(t, u_{2}, v_{2}, \overline{u_{2}}, \overline{v_{2}}\right)\right| \leq L_{g}\left(\left|u_{1}-u_{2}\right|+\left|\overline{u_{1}}-\overline{u_{2}}\right|+\left|v_{1}-v_{2}\right|+\left|\overline{v_{1}}-\overline{v_{2}}\right|\right) . \\
& \forall t \in[0, T], u_{1}, u_{2}, v_{1}, v_{2}, \overline{u_{1}}, \overline{u_{2}}, \overline{v_{1}}, \overline{v_{2}} \in \mathbb{R} .
\end{aligned}
$$

(A3) There exist real numbers $k_{1}, k_{2}, \tau_{1}, \tau_{2}, \varsigma_{1}, \varsigma_{2}$, $\zeta_{1}, \zeta_{2}, \xi_{1}, \xi_{2} \geq 0, \quad$ such that $|f(t, u, v, \bar{u}, \bar{v})| \leq$ $\left(k_{1}+\tau_{1}|u|+\varsigma_{1}|v|+\zeta_{1}|\bar{u}|+\xi_{1}|\bar{v}|\right)$,

$$
|g(t, u, v, \bar{u}, \bar{v})| \leq\left(k_{2}+\tau_{2}|u|+\varsigma_{2}|v|+\zeta_{2}|\bar{u}|+\xi_{1}|\bar{v}|\right), \quad \forall(t, u, v, \bar{u}, \bar{v}) \in[0, T] \times \mathbb{R}^{4}
$$

(A4) $\exists, W_{\alpha}, W_{\beta}, W_{\alpha, \beta} \in \mathbb{R}^{+}$, such that

$$
\begin{aligned}
& W_{\alpha}=\left[\left(\tau_{1}+\zeta_{1}\right)(Q+\mathscr{R})+\left(\tau_{2}+\zeta_{2}\right)\left(Q^{*}+\mathscr{R}^{*}\right)\right], \\
& W_{\beta}=\left[\left(\varsigma_{1}+\xi_{1}\right)(Q+\mathscr{R})+\left(\varsigma_{2}+\xi_{2}\right)\left(Q^{*}+\mathscr{R}^{*}\right)\right],
\end{aligned}
$$

with $\quad W_{\alpha, \beta}=\min \left\{1-\left[\left(\tau_{1}+\zeta_{1}\right)(Q+\mathscr{R})+\left(\tau_{2}+\zeta_{2}\right)\left(Q^{*}+\right.\right.\right.$ $\left.\left.\left.\mathscr{R}^{*}\right)\right], 1-\left[\left(\varsigma_{1}+\xi_{1}\right)(Q+\mathscr{R})+\left(\varsigma_{2}+\xi_{2}\right)\left(Q^{*}+\mathscr{R}^{*}\right)\right]\right\}$

$$
\begin{aligned}
\left|\mathscr{F}_{1}(u, v)(t)\right|= & \int_{0}^{t}\left|K_{1}(t, \tau)\right|\left|f\left(\tau, u(\tau), v(x),{ }^{c} D^{\alpha-1} u(\tau),{ }^{c} D^{\beta-1} v(\tau)\right)\right| \mathrm{d} \tau \\
& +\left|\omega_{1}(t)\right| \int_{0}^{T}\left|g\left(\tau, u(\tau), v(x),{ }^{c} D^{\alpha-1} u(\tau),{ }^{c} D^{\beta-1} v(\tau)\right)\right| \mathrm{d} \tau+\left|\omega_{2}(t)\right| \int_{0}^{T}\left|f\left(\tau, u(\tau), v(x),{ }^{c} D^{\alpha-1} u(\tau),{ }^{c} D^{\beta-1} v(\tau)\right)\right| \mathrm{d} \tau \\
& +\left|\omega_{3}(t)\right| \int_{0}^{\eta}\left|f\left(\tau, u(\tau), v(x),{ }^{c} D^{\alpha-1} u(\tau),{ }^{c} D^{\beta-1} v(\tau)\right)\right| \mathrm{d} \tau+\left|\omega_{4}(t)\right| \int_{0}^{\eta}\left|g\left(\tau, u(\tau), v(x),{ }^{c} D^{\alpha-1} u(\tau),{ }^{c} D^{\beta-1} v(\tau)\right)\right| \mathrm{d} \tau \\
& +\left|\omega_{5}(t)\right| \int_{0}^{T}\left|K_{2}(T, \tau)\right|\left|g\left(\tau, u(\tau), v(x),{ }^{c} D^{\alpha-1} u(\tau),{ }^{c} D^{\beta-1} v(\tau)\right)\right| \mathrm{d} \tau \\
& +\left|\omega_{6}(t)\right| \int_{0}^{T}\left|G_{2}(T, \tau)\right|\left|f\left(\tau, u(\tau), v(x),{ }^{c} D^{\alpha-1} u(\tau),{ }^{c} D^{\beta-1} v(\tau)\right)\right| \mathrm{d} \tau \\
& +\left|\omega_{7}(t)\right| \int_{0}^{\eta}\left|K_{2}(\eta, \tau)\right|\left|f\left(\tau, u(\tau), v(x),{ }^{c} D^{\alpha-1} u(\tau),{ }^{c} D^{\beta-1} v(\tau)\right)\right| \mathrm{d} \tau \\
& +\left|\omega_{8}(t)\right| \int_{0}^{\eta}\left|G_{2}(\eta, \tau)\right|\left|g\left(\tau, u(\tau), v(x),{ }^{c} D^{\alpha-1} u(\tau),{ }^{c} D^{\beta-1} v(\tau)\right)\right| \mathrm{d} \tau \\
& +\left|M_{1}\right| \int_{0}^{T}\left|G_{1}(T, \tau)\right|\left|g\left(\tau, u(\tau), v(x),{ }^{c} D^{\alpha-1} u(\tau),{ }^{c} D^{\beta-1} v(\tau)\right)\right| \mathrm{d} \tau \\
& +\left|M_{2}\right| \int_{0}^{\eta}\left|K_{1}(\eta, \tau) \| f\left(\tau, u(\tau), v(x),{ }^{c} D^{\alpha-1} u(\tau),{ }^{c} D^{\beta-1} v(\tau)\right)\right| \mathrm{d} \tau .
\end{aligned}
$$

Theorem 1. If both assumptions (A1) and (A2) are satisfied, and $\left(L_{f} \mathscr{R}+L_{f} Q+L_{g} \mathscr{R}^{*}+L_{g} Q^{*}\right)<1$, then the problem (1) Proof. Define a closed ball $\mathfrak{B}_{r}=\left\{(u, v) \in U_{\alpha-1} \times V_{\beta-1}\right.$ : $\left.\|(u, v)\|_{\alpha-1, \beta-1} \leq r\right\}$ with $r \geq N_{f}(\mathscr{R}+Q)+N_{g}\left(\mathscr{R}^{*}+Q^{*}\right) / 1-$ $\left(L_{f}(\mathscr{R}+Q)+L_{g}\left(\mathscr{R}^{*}+Q^{*}\right)\right)$, where $N_{f}=\sup _{0 \leq t \leq T} \mid f(t$,

First, we prove that $\mathscr{F} \mathfrak{B}_{r} \subset \mathfrak{B}_{r}, \forall(u, v) \in \mathfrak{B}_{r}, \forall t \in[0, T]$; we have, 


$$
\begin{aligned}
& \left|f\left(t, u(t), v(t),{ }^{c} D^{\alpha-1} u(t),{ }^{c} D^{\beta-1} v(t)\right)\right|=\left|f\left(t, u(t), v(t),{ }^{c} D^{\alpha-1} u(t),{ }^{c} D^{\beta-1} v(t)\right)-f(t, 0,0,0,0)+f(t, 0,0,0,0)\right| \\
\leq & \left|f\left(t, u(t), v(t),{ }^{c} D^{\alpha-1} u(t),{ }^{c} D^{\beta-1} v(t)\right)-f(t, 0,0,0,0)\right|+|f(t, 0,0,0,0)| \\
\leq & L_{f}\|(u, v)\|_{\alpha-1, \beta-1}+\sup _{0 \leq t \leq T}|f(t, 0,0,0,0)| \leq L_{f} r+N_{f} .
\end{aligned}
$$

Similarly,

$$
\left|g\left(t, u(t), v(t),{ }^{c} D^{\alpha-1} u(t),{ }^{c} D^{\beta-1} v(t)\right)\right| \leq L_{g} r+N_{g} .
$$

Then,

$$
\begin{aligned}
\left\|\mathscr{F}_{1}(u, v)\right\|= & \left(L_{f} r+N_{f}\right) \times\left[\sup _{0 \leq t \leq T} \int_{0}^{t}\left|K_{1}(t, \tau)\right| \mathrm{d} \tau+T\left\|\omega_{2}\right\|+\eta\left\|\omega_{3}\right\|+\left\|\omega_{6}\right\| \int_{0}^{T}\left|G_{2}(T, \tau)\right| \mathrm{d} \tau+\left\|\omega_{7}\right\| \int_{0}^{\eta}\left|K_{2}(\eta, \tau)\right| \mathrm{d} \tau\right. \\
& \left.+\left|M_{2}\right| \int_{0}^{\eta}\left|K_{1}(\eta, \tau)\right| \mathrm{d} \tau\right] \\
& +\left(L_{g} r+N_{g}\right) \times\left[T\left\|\omega_{1}\right\|+\eta\left\|\omega_{4}\right\|+\left\|\omega_{5}\right\| \int_{0}^{T}\left|K_{2}(T, \tau)\right| \mathrm{d} \tau+\left\|\omega_{8}\right\| \int_{0}^{\eta}\left|G_{2}(\eta, \tau)\right| \mathrm{d} \tau+\left|M_{1}\right| \int_{0}^{T}\left|G_{1}(T, \tau)\right| \mathrm{d} \tau\right] \\
\leq & \left(L_{f} r+N_{f}\right) Q_{1}+\left(L_{g} r+N_{g}\right) Q_{1}^{*},
\end{aligned}
$$

$$
\begin{aligned}
\left\|{ }^{c} D^{\alpha-1} \mathscr{F}_{1}(u, v)\right\|= & \left(L_{f} r+N_{f}\right) \times\left[\sup _{0 \leq t \leq T} \int_{0}^{t} \mathrm{~d} \tau+\lambda \int_{0}^{t}\left|K_{2}(t, \tau)\right| \mathrm{d} \tau+T\left\|{ }^{c} D^{\alpha-1} \omega_{2}\right\|+\eta\left\|^{c} D^{\alpha-1} \omega_{3}\right\|\right. \\
& \left.+\left\|{ }^{c} D^{\alpha-1} \omega_{6}\right\| \int_{0}^{T}\left|G_{2}(T, \tau)\right| d \tau+\left\|{ }^{c} D^{\alpha-1} \omega_{7}\right\| \int_{0}^{\eta}\left|K_{2}(\eta, \tau)\right| d \tau\right] \\
& +\left(L_{g} r+N_{g}\right) \times\left[T\|\|^{c} D^{\alpha-1} \omega_{1}\|+\eta\|{ }^{c} D^{\alpha-1} \omega_{4}\|+\|{ }^{c} D^{\alpha-1} \omega_{5}\left\|\int_{0}^{T}\left|K_{2}(T, \tau)\right| \mathrm{d} \tau+\right\|{ }^{c} D^{\alpha-1} \omega_{8} \| \int_{0}^{\eta}\left|G_{2}(\eta, \tau)\right| \mathrm{d} \tau\right], \\
\leq & \left(L_{f} r+N_{f}\right) Q_{2}+\left(L_{g} r+N_{g}\right) Q_{2}^{*} .
\end{aligned}
$$

(34) and (35) imply

$$
\left\|\mathscr{F}_{1}(u, v)\right\|_{\alpha-1} \leq\left(L_{f} r+N_{f}\right)\left(Q_{1}+Q_{2}\right)+\left(L_{g} r+N_{g}\right)\left(Q_{1}^{*}+Q_{2}^{*}\right) \text {. }
$$

$$
\begin{aligned}
\left\|\mathscr{F}_{2}(u, v)\right\|_{\beta-1} \leq & \left(L_{f} r+N_{f}\right)\left(\mathscr{R}_{1}+\mathscr{R}_{2}\right) \\
& +\left(L_{g} r+N_{g}\right)\left(\mathscr{R}_{1}^{*}+\mathscr{R}_{2}^{*}\right) .
\end{aligned}
$$

Similarly,

$$
\begin{aligned}
\|\mathscr{F}(u, v)\|_{\alpha-1, \beta-1} & \leq\left(L_{f} r+N_{f}\right)(\mathscr{R}+Q)+\left(L_{g} r+N_{g}\right)\left(\mathscr{R}^{*}+Q^{*}\right) \\
& =\left(L_{f} \mathscr{R}+L_{f} Q+L_{g} \mathscr{R}^{*}+L_{g} Q^{*}\right) r+N_{f} \mathscr{R}+N_{f} Q+N_{g} \mathscr{R}^{*}+N_{g} Q^{*} \leq r .
\end{aligned}
$$

That is, $\mathscr{F} \mathfrak{B}_{r} \subset \mathfrak{B}_{r}$. 
Now, we show that the operator $\mathscr{F}$ is a contraction. $\forall\left(u_{1}, v_{1}\right),\left(u_{2}, v_{2}\right) \in \Omega$. We have

$$
\begin{aligned}
& \left|\mathscr{F}_{1}\left(u_{1}, v_{1}\right)(t)-\mathscr{F}_{1}\left(u_{2}, v_{2}\right)(t)\right| \\
& \leq \int_{0}^{t}\left|K_{1}(t, \tau)\right|\left|f\left(\tau, u_{1}(\tau), v_{1}(\tau),{ }^{c} D^{\alpha-1} u_{1}(\tau),{ }^{c} D^{\beta-1} v_{1}(\tau)\right)-f\left(\tau, u_{2}(\tau), v_{2}(\tau),{ }^{c} D^{\alpha-1} u_{2}(\tau),{ }^{c} D^{\beta-1} v_{2}(\tau)\right)\right| \mathrm{d} \tau \\
& +\left\|\omega_{1}\right\| \int_{0}^{T}\left|g\left(\tau, u_{1}(\tau), v_{1}(\tau),{ }^{c} D^{\alpha-1} u_{1}(\tau),{ }^{c} D^{\beta-1} v_{1}(\tau)\right)-g\left(\tau, u_{2}(\tau), v_{2}(\tau),{ }^{c} D^{\alpha-1} u_{2}(\tau),{ }^{c} D^{\beta-1} v_{2}(\tau)\right)\right| \mathrm{d} \tau \\
& +\left\|\omega_{2}\right\| \int_{0}^{T}\left|f\left(\tau, u_{1}(\tau), v_{1}(\tau),{ }^{c} D^{\alpha-1} u_{1}(\tau),{ }^{c} D^{\beta-1} v_{1}(\tau)\right)-f\left(\tau, u_{2}(\tau), v_{2}(\tau),{ }^{c} D^{\alpha-1} u_{2}(\tau),{ }^{c} D^{\beta-1} v_{2}(\tau)\right)\right| \mathrm{d} \tau \\
& +\left\|\omega_{3}\right\| \int_{0}^{\eta}\left|f\left(\tau, u_{1}(\tau), v_{1}(\tau),{ }^{c} D^{\alpha-1} u_{1}(\tau),{ }^{c} D^{\beta-1} v_{1}(\tau)\right)-f\left(\tau, u_{2}(\tau), v_{2}(\tau),{ }^{c} D^{\alpha-1} u_{2}(\tau),{ }^{c} D^{\beta-1} v_{2}(\tau)\right)\right| \mathrm{d} \tau \\
& +\left\|\omega_{4}\right\| \int_{0}^{\eta}\left|g\left(\tau, u_{1}(\tau), v_{1}(\tau),{ }^{c} D^{\alpha-1} u_{1}(\tau),{ }^{c} D^{\beta-1} v_{1}(\tau)\right)-g\left(\tau, u_{2}(\tau), v_{2}(\tau),{ }^{c} D^{\alpha-1} u_{2}(\tau),{ }^{c} D^{\beta-1} v_{2}(\tau)\right)\right| \mathrm{d} \tau \\
& +\left\|\omega_{5}\right\| \int_{0}^{T}\left|K_{2}(T, \tau) \| g\left(\tau, u_{1}(\tau), v_{1}(\tau),{ }^{c} D^{\alpha-1} u_{1}(\tau),{ }^{c} D^{\beta-1} v_{1}(\tau)\right)-g\left(\tau, u_{2}(\tau), v_{2}(\tau),{ }^{c} D^{\alpha-1} u_{2}(\tau),{ }^{c} D^{\beta-1} v_{2}(\tau)\right)\right| \mathrm{d} \tau \\
& +\left\|\omega_{6}\right\| \int_{0}^{T}\left|G_{2}(T, \tau) \| f\left(\tau, u_{1}(\tau), v_{1}(\tau),{ }^{c} D^{\alpha-1} u_{1}(\tau),{ }^{c} D^{\beta-1} v_{1}(\tau)\right)-f\left(\tau, u_{2}(\tau), v_{2}(\tau),{ }^{c} D^{\alpha-1} u_{2}(\tau),{ }^{c} D^{\beta-1} v_{2}(\tau)\right)\right| \mathrm{d} \tau \\
& +\left\|\omega_{7}\right\| \int_{0}^{\eta}\left|K_{2}(\eta, \tau) \| f\left(\tau, u_{1}(\tau), v_{1}(\tau),{ }^{c} D^{\alpha-1} u_{1}(\tau),{ }^{c} D^{\beta-1} v_{1}(\tau)\right)-f\left(\tau, u_{2}(\tau), v_{2}(\tau),{ }^{c} D^{\alpha-1} u_{2}(\tau),{ }^{c} D^{\beta-1} v_{2}(\tau)\right)\right| \mathrm{d} \tau \\
& +\left\|\omega_{8}\right\| \int_{0}^{\eta}\left|G_{2}(\eta, \tau) \| g\left(\tau, u_{1}(\tau), v_{1}(\tau),{ }^{c} D^{\alpha-1} u_{1}(\tau),{ }^{c} D^{\beta-1} v_{1}(\tau)\right)-g\left(\tau, u_{2}(\tau), v_{2}(\tau),{ }^{c} D^{\alpha-1} u_{2}(\tau),{ }^{c} D^{\beta-1} v_{2}(\tau)\right)\right| \mathrm{d} \tau \\
& +\left|M_{1}\right| \int_{0}^{T}\left|G_{1}(T, \tau)\right|\left|g\left(\tau, u_{1}(\tau), v_{1}(\tau),{ }^{c} D^{\alpha-1} u_{1}(\tau),{ }^{c} D^{\beta-1} v_{1}(\tau)\right)-g\left(\tau, u_{2}(\tau), v_{2}(\tau),{ }^{c} D^{\alpha-1} u_{2}(\tau),{ }^{c} D^{\beta-1} v_{2}(\tau)\right)\right| \mathrm{d} \tau \\
& +\left|M_{2}\right| \int_{0}^{\eta}\left|K_{1}(\eta, \tau)\right|\left|f\left(\tau, u_{1}(\tau), v_{1}(\tau),{ }^{c} D^{\alpha-1} u_{1}(\tau),{ }^{c} D^{\beta-1} v_{1}(\tau)\right)-f\left(\tau, u_{2}(\tau), v_{2}(\tau),{ }^{c} D^{\alpha-1} u_{2}(\tau),{ }^{c} D^{\beta-1} v_{2}(\tau)\right)\right| \mathrm{d} \tau, \\
& \leq L_{f} Q_{1}\left(\left\|u_{1}-u_{2}\right\|_{\alpha-1, \beta-1}+\left\|v_{1}-v_{2}\right\|_{\alpha-1, \beta-1}\right)+L_{g} Q_{1}^{*}\left(\left\|u_{1}-u_{2}\right\|_{\alpha-1, \beta-1}+\left\|v_{1}-v_{2}\right\|_{\alpha-1, \beta-1}\right) .
\end{aligned}
$$

One can easily show

$\left\|{ }^{c} D^{\alpha-1} \mathscr{F}_{1}\left(u_{1}, v_{1}\right)-{ }^{c} D^{\alpha-1} \mathscr{F}_{1}\left(u_{2}, v_{2}\right)\right\| \leq L_{f} Q_{2}\left(\left\|u_{1}-u_{2}\right\|_{\alpha-1, \beta-1}+\left\|v_{1}-v_{2}\right\|_{\alpha-1, \beta-1}\right)+L_{g} Q_{2}^{*}\left(\left\|u_{1}-u_{2}\right\|_{\alpha-1, \beta-1}+\left\|v_{1}-v_{2}\right\|_{\alpha-1, \beta-1}\right)$.

Combining (40) and (41) leads to

$$
\left\|\mathscr{F}_{1}\left(u_{1}, v_{1}\right)-\mathscr{F}_{1}\left(u_{2}, v_{2}\right)\right\|_{\alpha-1} \leq\left(L_{f} Q+L_{g} Q^{*}\right)\left(\left\|u_{1}-u_{2}\right\|_{\alpha-1, \beta-1}+\left\|v_{1}-v_{2}\right\|_{\alpha-1, \beta-1}\right)
$$

Similarly, 


$$
\left\|\mathscr{F}_{2}\left(u_{1}, v_{1}\right)-\mathscr{F}_{2}\left(u_{2}, v_{2}\right)\right\|_{\beta-1} \leq\left(L_{f} \mathscr{R}+L_{g} \mathscr{R}^{*}\right)\left(\left\|u_{1}-u_{2}\right\|_{\alpha-1, \beta-1}+\left\|v_{1}-v_{2}\right\|_{\alpha-1, \beta-1}\right) .
$$

By (42) and (43), we have

$$
\left\|\mathscr{F}\left(u_{1}, v_{1}\right)-\mathscr{F}\left(u_{2}, v_{2}\right)\right\|_{\alpha-1, \beta-1} \leq\left(L_{f} Q+L_{g} Q^{*}+L_{f} \mathscr{R}+L_{g} \mathscr{R}^{*}\right)\left(\left\|u_{1}-u_{2}\right\|_{\alpha-1, \beta-1}+\left\|v_{1}-v_{2}\right\|_{\alpha-1, \beta-1}\right),
$$

which shows that the operator is a contraction and the proof is completed.

Theorem 2. Suppose the assumptions (A1) and (A3) are satisfied and assume that from (A4) that $W_{\alpha}<1$ and $W_{\beta}<1$. Then, problem (1) has a solution on $[0, T]$.

Proof. First, we show that the operator $\mathscr{F}: \Omega \longrightarrow \Omega$ is completely continuous. $\mathscr{F}$ is continuous as a result of the continuity of $f$ and $g$.

Define $S$ to be a bounded set in $\Omega$. Then, there exist positive constants $l_{1}, l_{2}$ such that $|f(\cdot, u(\cdot), v(\cdot), \bar{u}(\cdot), \bar{v}(\cdot))|$ $\leq l_{1},|g(\cdot, u(\cdot), v(\cdot), \bar{u}(\cdot), \bar{v}(\cdot))| \leq l_{2}, \forall(u, v, \bar{u}, \bar{v}) \in S$.

Then, for any $(u, v, \bar{u}, \bar{v}) \in S$, it follows that

$$
\begin{array}{r}
\left\|\mathscr{F}_{1}(u, v)\right\| \leq l_{1} Q_{1}+l_{2} Q_{1}^{*}, \\
\left\|{ }^{c} D^{\alpha-1} \mathscr{F}_{1}(u, v)\right\| \leq l_{1} \mathscr{R}_{1}+l_{2} \mathscr{R}_{1}^{*} .
\end{array}
$$

\section{Combining (45) and (46), we obtain}

$$
\left\|\mathscr{F}_{1}(u, v)\right\|_{\alpha-1} \leq l_{1}\left(Q_{1}+\mathscr{R}_{1}\right)+l_{2}\left(Q_{1}^{*}+\mathscr{R}_{1}^{*}\right) .
$$

Similarly,

$$
\left\|\mathscr{F}_{2}(u, v)\right\|_{\beta-1} \leq l_{1}\left(Q_{2}+\mathscr{R}_{2}\right)+l_{2}\left(Q_{2}^{*}+\mathscr{R}_{2}^{*}\right) .
$$

(47) and (48) imply

$$
\|\mathscr{F}(u, v)\|_{\alpha-1, \beta-1} \leq l_{1}(Q+\mathscr{R})+l_{2}\left(Q^{*}+\mathscr{R}^{*}\right) .
$$

By equation (49), we showed the operator $\mathscr{F}$ is uniformly bounded.

Next, we show that $\mathscr{F}$ is equicontinuous; let $t_{1}, t_{2} \in[0, T]$ with $t_{1}<t_{2}$, then we have,

$$
\begin{aligned}
& \left|\mathscr{F}_{1}(u, v)\left(t_{2}\right)-\mathscr{F}_{1}(u, v)\left(t_{1}\right)\right| \leq \\
& \left|\begin{array}{l}
\int_{0}^{t_{2}} K_{1}\left(t_{2}, \tau\right) f\left(\tau, u(\tau), v(\tau),{ }^{c} D^{\alpha-1} u(\tau),{ }^{c} D^{\beta-1} v(\tau)\right) d \tau \\
-\int_{0}^{t_{1}} K_{1}\left(t_{1}, \tau\right) f\left(\tau, u(\tau), v(\tau),{ }^{c} D^{\beta-1} u(\tau),{ }^{c} D^{\beta-1} v(\tau)\right) \mathrm{d} \tau
\end{array}\right| \\
& +\left|\omega_{1}\left(t_{2}\right)-\omega_{1}\left(t_{1}\right)\right| \int_{0}^{T}\left|g\left(\tau, u(\tau), v(\tau),{ }^{c} D^{\alpha-1} u(\tau),{ }^{c} D^{\beta-1} v(\tau)\right)\right| \mathrm{d} \tau \\
& +\left|\omega_{2}\left(t_{2}\right)-\omega_{2}\left(t_{1}\right)\right| \int_{0}^{T}\left|f\left(\tau, u(\tau), v(\tau),{ }^{c} D^{\alpha-1} u(\tau),{ }^{c} D^{\beta-1} v(\tau)\right)\right| \mathrm{d} \tau \\
& +\left|\omega_{3}\left(t_{2}\right)-\omega_{3}\left(t_{1}\right)\right| \int_{0}^{\eta}\left|f\left(\tau, u(\tau), v(\tau),{ }^{c} D^{\alpha-1} u(\tau),{ }^{c} D^{\beta-1} v(\tau)\right)\right| \mathrm{d} \tau \\
& +\left|\omega_{4}\left(t_{2}\right)-\omega_{4}\left(t_{1}\right)\right| \int_{0}^{\eta}\left|g\left(\tau, u(\tau), v(\tau),{ }^{c} D^{\alpha-1} u(\tau),{ }^{c} D^{\beta-1} v(\tau)\right)\right| \mathrm{d} \tau \\
& +\left|\omega_{5}\left(t_{2}\right)-\omega_{5}\left(t_{1}\right)\right| \int_{0}^{T}\left|K_{2}(T, \tau)\right|\left|g\left(\tau, u(\tau), v(\tau),{ }^{c} D^{\alpha-1} u(\tau),{ }^{c} D^{\beta-1} v(\tau)\right)\right| \mathrm{d} \tau \\
& +\left|\omega_{6}\left(t_{2}\right)-\omega_{6}\left(t_{1}\right)\right| \int_{0}^{T}\left|G_{2}(T, \tau)\right|\left|f\left(\tau, u(\tau), v(\tau),{ }^{c} D^{\alpha-1} u(\tau),{ }^{c} D^{\beta-1} v(\tau)\right)\right| \mathrm{d} \tau \\
& +\left|\omega_{7}\left(t_{2}\right)-\omega_{7}\left(t_{1}\right)\right| \int_{0}^{\eta}\left|K_{2}(\eta, \tau)\right|\left|f\left(\tau, u(\tau), v(\tau),{ }^{c} D^{\alpha-1} u(\tau),{ }^{c} D^{\beta-1} v(\tau)\right)\right| \mathrm{d} \tau \\
& +\left|\omega_{8}\left(t_{2}\right)-\omega_{8}\left(t_{1}\right)\right| \int_{0}^{\eta}\left|G_{2}(\eta, \tau)\right|\left|g\left(\tau, u(\tau), v(\tau),{ }^{c} D^{\alpha-1} u(\tau),{ }^{c} D^{\beta-1} v(\tau)\right)\right| \mathrm{d} \tau \text {. }
\end{aligned}
$$


Then, the R.H.S. of (50) approaches zero as $t_{1} \longrightarrow t_{2}$ and implies $\left|\mathscr{F}_{1}(u, v)\left(t_{2}\right)-\mathscr{F}_{1}(u, v)\left(t_{1}\right)\right| \longrightarrow 0$ as $t_{1} \longrightarrow t_{2}$.

In a like manner,

$\left|{ }^{c} D^{\alpha-1} \mathscr{F}_{1}(u, v)\left(t_{2}\right)-{ }^{c} D^{\alpha-1} \mathscr{F}_{1}(u, v)\left(t_{1}\right)\right| \longrightarrow 0, \quad$ as $t_{1} \longrightarrow t_{2}$.

Also, one can easily show that, $\mid \mathscr{F}_{2}(u, v)\left(t_{2}\right)-$ $\mathscr{F}_{2}(u, v)\left(t_{1}\right) \mid \longrightarrow 0$ and $\mid{ }^{c} D^{\beta-1} \mathscr{F}_{2}(u, v)\left(t_{2}\right)-{ }^{c} D^{\beta-1} \mathscr{F}_{2}$ $(u, v)\left(t_{1}\right) \mid \longrightarrow 0, \quad$ as $t_{1} \longrightarrow t_{2}$.
Accordingly, $\mathscr{F}(u, v)$ is equicontinuous, and so $\mathscr{F}$ is completely continuous.

Finally, we need to prove that the set $X=\{(u, v)$ $\in \Omega:(u, v)=\theta \mathscr{F}(u, v), \theta \in[0,1]\}$ is bounded,

$\forall t \in[0, T], \forall(u, v) \in X$, we have.

$u(t)=\theta \mathscr{F}_{1}(u, v)$, and $v(t)=\theta \mathscr{F}_{2}(u, v)$, then

$$
\begin{aligned}
|u(t)| & \leq\left(k_{1}+\tau_{1}|u|+\varsigma_{1}|v|+\zeta_{1}|\bar{u}|+\xi_{1}|\bar{v}|\right) Q_{1}+\left(k_{2}+\tau_{2}|u|+\varsigma_{2}|v|+\zeta_{2}|\bar{u}|+\xi_{2}|\bar{v}|\right) Q_{1}^{*} \\
& =k_{1} Q_{1}+k_{2} Q_{1}^{*}+\left(\tau_{1} Q_{1}+\tau_{2} Q_{1}^{*}\right)|u|+\left(\varsigma_{1} Q_{1}+\varsigma_{2} Q_{1}^{*}\right)|v|+\left(\zeta_{1} Q_{1}+\zeta_{2} Q_{1}^{*}\right)|\bar{u}|+\left(\xi_{1} Q_{1}+\xi_{2} Q_{1}^{*}\right)|\bar{v}|, \\
\left|c D^{\alpha-1} u(t)\right| & \leq\left(k_{1}+\tau_{1}|u|+\varsigma_{1}|v|+\zeta_{1}|\bar{u}|+\xi_{1}|\bar{v}|\right) \mathscr{R}_{1}+\left(k_{2}+\tau_{2}|u|+\varsigma_{2}|v|+\zeta_{2}|\bar{u}|+\xi_{2}|\bar{v}|\right) \mathscr{R}_{1}^{*} \\
& =k_{1} \mathscr{R}_{1}+k_{2} \mathscr{R}_{1}^{*}+\left(\tau_{1} \mathscr{R}_{1}+\tau_{2} \mathscr{R}_{1}^{*}\right)|u|+\left(\varsigma_{1} \mathscr{R}_{1}+\varsigma_{2} \mathscr{R}_{1}^{*}\right)|v|+\left(\zeta_{1} \mathscr{R}_{1}+\zeta_{2} \mathscr{R}_{1}^{*}\right)|\bar{u}|+\left(\xi_{1} \mathscr{R}_{1}+\xi_{2} \mathscr{R}_{1}^{*}\right)|\bar{v}| .
\end{aligned}
$$

Hence, we have,

$$
\begin{aligned}
\|u\|_{\alpha-1} \leq & k_{1}\left(Q_{1}+\mathscr{R}_{1}\right)+k_{2}\left(Q_{1}^{*}+\mathscr{R}_{1}^{*}\right)+\left[\tau_{1}\left(Q_{1}+\mathscr{R}_{1}\right)+\tau_{2}\left(Q_{1}^{*}+\mathscr{R}_{1}^{*}\right)\right]\|u\|+\left[\varsigma_{1}\left(Q_{1}+\mathscr{R}_{1}\right)+\varsigma_{2}\left(Q_{1}^{*}+\mathscr{R}_{1}^{*}\right)\right]\|v\| \\
& +\left[\zeta_{1}\left(Q_{1}+\mathscr{R}_{1}\right)+\zeta_{2}\left(Q_{1}^{*}+\mathscr{R}_{1}^{*}\right)\right]\|\bar{u}\|+\left[\xi_{1}\left(Q_{1}+\mathscr{R}_{1}\right)+\xi_{2}\left(Q_{1}^{*}+\mathscr{R}_{1}^{*}\right)\right]\|\bar{v}\|, \\
\|v\|_{\beta-1} \leq & k_{1}\left(Q_{2}+\mathscr{R}_{2}\right)+k_{2}\left(Q_{2}^{*}+\mathscr{R}_{2}^{*}\right)+\left[\tau_{1}\left(Q_{2}+\mathscr{R}_{2}\right)+\tau_{2}\left(Q_{2}^{*}+\mathscr{R}_{2}^{*}\right)\right]\|u\|+\left[\varsigma_{1}\left(Q_{2}+\mathscr{R}_{2}\right)+\varsigma_{2}\left(Q_{2}^{*}+\mathscr{R}_{2}^{*}\right)\right]\|v\| \\
& +\left[\zeta_{1}\left(Q_{2}+\mathscr{R}_{2}\right)+\zeta_{2}\left(Q_{2}^{*}+\mathscr{R}_{2}^{*}\right)\right]\|\bar{u}\|+\left[\xi_{1}\left(Q_{2}+\mathscr{R}_{2}\right)+\xi_{2}\left(Q_{2}^{*}+\mathscr{R}_{2}^{*}\right)\right]\|\bar{v}\|,
\end{aligned}
$$

which imply that

$$
\begin{aligned}
\|u\|_{\alpha-1}+\|v\|_{\beta-1} \leq & k_{1}(Q+\mathscr{R})+k_{2}\left(Q^{*}+\mathscr{R}^{*}\right)+\left[\tau_{1}(Q+\mathscr{R})+\tau_{2}\left(Q^{*}+\mathscr{R}^{*}\right)\right]\|u\|+\left[\varsigma_{1}(Q+\mathscr{R})+\varsigma_{2}\left(Q^{*}+\mathscr{R}^{*}\right)\right]\|v\| \\
& +\left[\zeta_{1}(Q+\mathscr{R})+\zeta_{2}\left(Q^{*}+\mathscr{R}^{*}\right)\right]\|\bar{u}\|+\left[\xi_{1}(Q+\mathscr{R})+\xi_{2}\left(Q^{*}+\mathscr{R}^{*}\right)\right]\|\bar{v}\| .
\end{aligned}
$$

But,

Then, (55) becomes,

$$
\begin{aligned}
\|u\|_{\alpha-1} & =\|u\|+\|\bar{u}\|, \\
\|v\|_{\beta-1} & =\|v\|+\|\bar{v}\| .
\end{aligned}
$$

$$
\begin{aligned}
\|u\|_{\alpha-1}+\|v\|_{\beta-1} \leq & k_{1}(Q+\mathscr{R})+k_{2}\left(Q^{*}+\mathscr{R}^{*}\right)+\left[\left(\tau_{1}+\zeta_{1}\right)(Q+\mathscr{R})+\left(\tau_{2}+\zeta_{2}\right)\left(Q^{*}+\mathscr{R}^{*}\right)\right]\|u\|_{\alpha-1} \\
& +\left[\left(\varsigma_{1}+\xi_{1}\right)(Q+\mathscr{R})+\left(\varsigma_{2}+\xi_{2}\right)\left(Q^{*}+\mathscr{R}^{*}\right)\right]\|v\|_{\beta-1} .
\end{aligned}
$$

Consequently,

$$
\|(u, v)\|_{\alpha-1, \beta-1} \leq \frac{k_{1}(Q+\mathscr{R})+k_{2}\left(Q^{*}+\mathscr{R}^{*}\right)}{W_{\alpha, \beta}}, \quad W_{\alpha, \beta} \neq 0,
$$


which shows the boundedness of $X$ is bounded. So, by Leray-Schauder's alternative, $\mathscr{F}$ has a fixed point, implying the existence of a solution for the BVP given by (1).

\section{Example}

Given the problem,

$$
\left\{\begin{array}{l}
{ }^{c} D^{1 / 2}(D+1) u(t)=\left(\frac{1}{9\left(36+t^{2}\right)}\right)\left(u(t)+\sin (v(t))+\left|{ }^{c} D^{1 / 2} u(t)\right|+\left|{ }^{c} D^{1 / 4} v(t)\right|\right), 0 \leq t \leq 3, \\
{ }^{c} D^{1 / 4}(D-1) v(t)=\left(\frac{1}{7\left(64-t^{4}\right)}\right)\left(\sin (u(t))+|v(t)|+\frac{\left|{ }^{c} D^{1 / 4} v(t)\right|}{1+\left|{ }^{c} D^{1 / 4} v(t)\right|}+\tan ^{-1}\left({ }^{c} D^{1 / 2} u(t)\right)\right), \\
u(1)=v(3),{ }^{c} D^{1 / 2} u(1)=+{ }^{c} D^{1 / 2} v(3), \\
v(1)=u(3),{ }^{c} D^{1 / 4} v(1)=+{ }^{c} D^{1 / 4} u(3) .
\end{array}\right.
$$

Here,

$$
\begin{aligned}
f\left(t, u(t), v(t),{ }^{c} D^{\alpha-1} u(t),{ }^{c} D^{\beta-1} v(t)\right) & =\left(\frac{1}{9\left(36+t^{2}\right)}\right)\left(u(t)+\sin (v(t))+\left|{ }^{c} D^{1 / 2} u(t)\right|+\left|{ }^{c} D^{1 / 4} v(t)\right|\right), \\
g\left(t, u(t), v(t),{ }^{c} D^{\alpha-1} u(t),{ }^{c} D^{\beta-1} v(t)\right) & =\left(\frac{1}{7\left(64-t^{4}\right)}\right)\left(\sin (u(t))+|v(t)|+\frac{\left|{ }^{c} D^{1 / 4} v(t)\right|}{1+\left|{ }^{c} D^{1 / 4} v(t)\right|}+\tan ^{-1}\left({ }^{c} D^{1 / 2} u(t)\right)\right), \\
\lambda & =1, \mu=-1, \alpha=\frac{3}{2},=\beta=\frac{5}{4}, \eta=1, T=3, \varepsilon_{1}=\varepsilon_{2}=1, \delta_{1}=2, \text { and } \delta_{2}=1,
\end{aligned}
$$

with

$$
\begin{aligned}
\rho_{1} & \leq 1.13, \rho_{2} \leq 39.31, q_{1} \leq 2.95, q_{2} \leq-12.84, \Delta_{1}=152.41, \Delta_{2}=-1, \\
M_{1} & =-1.1, M_{2}=-1.013, N_{1}=-2, \text { and } N_{2}=2 .
\end{aligned}
$$

We have,

$$
\begin{aligned}
\left\|\theta_{1}\right\| & =0.44,\left\|\theta_{2}\right\|=0.004,\left\|\theta_{3}\right\|=0.44,\left\|\theta_{4}\right\|=0.55,\left\|\theta_{5}\right\|=1.056,\left\|\theta_{6}\right\|=3.4,\left\|\theta_{7}\right\|=3.1,\left\|\theta_{8}\right\|=0.05, \text { and } \\
\left\|\omega_{1}\right\| & =0.17,\left\|\omega_{2}\right\|=0.03,\left\|\omega_{3}\right\|=0.19,\left\|\omega_{4}\right\|=0.44,\left\|\omega_{5}\right\|=69,\left\|\omega_{6}\right\|=0.5,\left\|\omega_{7}\right\|=0.17,\left\|\omega_{8}\right\|=0.58 .
\end{aligned}
$$

Clearly, the functions $f, g$ satisfy the hypotheses (A1) and (A2), from the inequalities, 


$$
\begin{aligned}
& \left|f\left(t, u_{1}(t), v_{1}(t),{ }^{c} D^{1 / 2} u_{1}(t),{ }^{c} D^{1 / 4} v_{1}(t)\right)-f\left(t, u_{2}(t), v_{2}(t),{ }^{c} D^{1 / 2} u_{2}(t),{ }^{c} D^{1 / 4} v_{2}(t)\right)\right| \\
\leq & \frac{1}{324}\left(\left|u_{1}(t)-u_{2}(t)\right|+\left|v_{1}(t)-v_{2}(t)\right|+\left|{ }^{c} D^{1 / 2} u_{1}(t)-{ }^{c} D^{1 / 2} u_{2}(t)\right|+\left|{ }^{c} D^{1 / 4} v_{1}(t)-{ }^{c} D^{1 / 4} v_{2}(t)\right|\right) . \\
& \left|g\left(t, u_{1}(t), v_{1}(t),{ }^{c} D^{1 / 2} u_{1}(t),{ }^{c} D^{1 / 4} v_{1}(t)\right)-g\left(t, u_{2}(t), v_{2}(t),{ }^{c} D^{1 / 2} u_{2}(t),{ }^{c} D^{1 / 4} v_{2}(t)\right)\right| \\
\leq & \frac{1}{448}\left(\left|u_{1}(t)-u_{2}(t)\right|+\left|v_{1}(t)-v_{2}(t)\right|+\left|{ }^{c} D^{1 / 2} u_{1}(t)-{ }^{c} D^{1 / 2} u_{2}(t)\right|+\left|{ }^{c} D^{1 / 4} v_{1}(t)-{ }^{c} D^{1 / 4} v_{2}(t)\right|\right),
\end{aligned}
$$

with

$$
\begin{aligned}
Q & =Q_{1}+Q_{2}, \\
Q^{*} & =Q_{1}^{*}+Q_{2}^{*}, \\
\mathscr{R} & =\mathscr{R}_{1}+\mathscr{R}_{2}, \\
\mathscr{R}^{*} & =\mathscr{R}_{1}^{*}+\mathscr{R}_{2}^{*}, \\
Q_{1} & \leq 12.54, \\
Q_{1}^{*} & \leq 23.93, \\
Q_{2} & \leq 10.087, \\
Q_{2}^{*} & \leq 6.2079, \\
\mathscr{R}_{1} & \leq 194.82, \\
\mathscr{R}_{1}^{*} & \leq 89.114, \\
\mathscr{R}_{2} & \leq 0.25, \\
\mathscr{R}_{2}^{*} & \leq 24.07 .
\end{aligned}
$$

Observe that, $\left(L_{f} \mathscr{R}+L_{f} Q+L_{g} \mathscr{R}^{*}+L_{g} Q^{*}\right)=(1 / 324$ $(195.07+22.627)+1 / 448(113.184+30.1379))=0.991814$ $<1$; Theorem 1 implies that our problem has unique solution.

\section{Conclusion and Future Work}

In this article, we investigate the existence result of the system of fractional differential equations given in problem (1). For the future work, the researcher may generalize our system by taking an $n \times 1$ system of sequential type and may apply another type of fractional derivatives such as Hadamard and Psi-Caputo fractional derivatives.

\section{Data Availability}

No data were used to support this study.

\section{Conflicts of Interest}

The author declares that he has no conflicts of interest.

\section{Acknowledgments}

This article was funded by the Deanship of Scientific Research (DSR), under NASHER track with a grant number (216043), King Faisal University (KFU), Ahsa, Saudi Arabia. The author, therefore, acknowledges technical and financial support of DSR at KFU.

\section{References}

[1] I. Podlubny, Fractional Differential Equations, Academic Press, Cambridge, MA, USA, 1999.

[2] A. A. Kilbas, H. M. Srivastava, and J. J. Trujillo, Theory and Applications of Fractional Differential Equations, Elsevier Science B.V., Amsterdam, Netherlands, 2006.

[3] R. Agarwal, D. O’Regan, and S. Hristova, "Stability of Caputo fractional differential equations by Lyapunov functions," Applications of Mathematics, vol. 60, no. 6, pp. 653-676, 2015.

[4] C. Bai, "Impulsive periodic boundary value problems for fractional differential equation involving Riemann-Liouville sequential fractional derivative," Journal of Mathematical Analysis and Applications, vol. 384, no. 2, pp. 211-231, 2011.

[5] B. Ahmad and J. J. Nieto, "Sequential fractional differential equations with three-point boundary conditions," Computers \& Mathematics with Applications, vol. 64, no. 10, pp. 3046-3052, 2012.

[6] B. Ahmad and J. J. Nieto, "Boundary value problems for a class of sequential integrodifferential equations of fractional order," J. Funct. Spaces Appl, vol. 2013, Article ID 149659, 8 pages, 2013.

[7] B. Ahmad and S. Ntouyas, "Existence results for a coupled system of Caputo type sequential fractional differential equations with nonlocal integral boundary conditions," Applied Mathematics and Computation, vol. 266, pp. 615-622, 2015.

[8] M. H. Aqlan, A. Alsaedi, A. Alsaedi, B. Ahmad, and J. J. Nieto, "Existence theory for sequential fractional differential equations with anti-periodic type boundary conditions," Open Mathematics, vol. 14, no. 1, pp. 723-735, 2016.

[9] M. Klimek, "Sequential fractional differential equations with Hadamard derivative," Communications in Nonlinear Science and Numerical Simulation, vol. 16, no. 12, pp. 4689-4697, 2011.

[10] H. Ye and R. Huang, "On the nonlinear fractional differential equations with Caputo sequential fractional derivative," $A d v$. Math. Phys, vol. 2015, Article ID 174156, 9 pages, 2015.

[11] A. Alsaedi, S. Sivasundaram, and B. Ahmad, "On the generalization of second order nonlinear anti-periodic boundary value problems," Nonlinear Studies, vol. 16, pp. 415-420, 2009.

[12] B. Ahmad and J. J. Nieto, "Anti-periodic fractional boundary value problems," Computers \& Mathematics with Applications, vol. 62, no. 3, pp. 1150-1156, 2011.

[13] B. Ahmad, J. Losada, and J. J. Nieto, "On antiperiodic nonlocal three-point boundary value problems for nonlinear fractional differential equations, Discrete Dyn. Nat," Forestry and Society, vol. 2015, Article ID 973783, 2015.

[14] L. Zhang, B. Ahmed, and G. Wang, "Existence and approximation of positive solutions for nonlinear fractional integrodifferential boundary value problems on an unbounded domain," Appl. Comput. Math, vol. 15, no. 2, pp. 149-158, 2016. 
[15] Y. Huangi, Z. Liu, and R. Wang, "Quasilinearization for higher order impulsive fractional differential equa-tions," Appl. Comput. Math, vol. 15, no. 2, pp. 159-171, 2016.

[16] J. Wang, W. Wei, and M. Fečkan, "Nonlocal Cauchy problems for fractional evolution equations involving Volterra-Fredholm type integral operators," Miskolc Mathematical Notes, vol. 13, no. 1, pp. 127-147, 2012.

[17] J. R. Wang, Y. Zhou, and M. Feckan, "On the nonlocal Cauchy problem for semilinear fractional order evolution equations," Central European Journal of Mathematics, vol. 12, pp. 911-922, 2104.

[18] M. Awadalla, Y. Y. Y. Noupoue, and K. A. Asbeh, "Psi-caputo logistic population growth model," Journal of Mathematics, vol. 2021, Article ID 8634280, 9 pages, 2021.

[19] M. Awadalla, Y. Y. Y. Noupoue, and K. Abuasbeh, "Population growth modeling via Rayleigh-caputo fractional derivative," Journal of Statistics Applications \& Probability, vol. 10, no. 1, 2021.

[20] M. Awadalla, Y. Y. N. Yannick, and K. A. Asbeh, "Modeling the dependence of barometric pressure with altitude using caputo and caputo-fabrizio fractional derivatives," Journal of Mathematics, vol. 2020, Article ID 2417681, 9 pages, 2020.

[21] N. I. Mahmudov, M. Awadalla, and K. Abuassba, "Nonlinear sequential fractional differential equations with nonlocal boundary conditions," Advances in Difference Equations, vol. 2017, no. 1, pp. 1-15, 2017.

[22] X. Su, "Boundary value problem for a coupled system of nonlinear fractional differential equations," Applied Mathematics Letters, vol. 22, no. 1, pp. 64-69, 2009.

[23] Y. Zhou, C. Zhang, and H. Wang, "Boundary value methods for Caputo fractional differential equations," Journal of Computational Mathematics, vol. 39, no. 1, pp. 108-129, 2021.

[24] B. Ahmad, Y. Alruwaily, A. Alsaedi, and S. K. Ntouyas, "Sequential fractional differential equations with nonlocal integro-multipoint boundary conditions," Novi Sad Journal of Mathematics Accepted, 2021, https://sites.dmi.uns.ac.rs/ nsjom/Papers/Accepted/ns12668.pdf.

[25] A. Wongcharoen, S. K. Ntouyas, P. Wongsantisuk, and J. Tariboon, "Existence results for a nonlocal coupled system of sequential fractional differential equations involving-hilfer fractional derivatives," Advances in Mathematical Physics, vol. 2021, Article ID 5554619, 9 pages, 2021.

[26] Z. Bouazza, S. Etemad, M. S. Souid, S. Rezapour, F. Martínez, and M. K. Kaabar, "A study on the solutions of a multiterm FBVP of variable order," Journal of Function Spaces, vol. 2021, Article ID 9939147, 9 pages, 2021. 